\title{
1 EHD2-mediated restriction of caveolar dynamics regulates cellular lipid uptake
}

3 Claudia Matthäus ${ }^{1,10^{*}}$, Ines Lahmann ${ }^{2,10}$, Séverine Kunz ${ }^{3}$, Wenke Jonas ${ }^{4}$, Arthur Alves Melo ${ }^{1}$, Martin

4 Lehmann $^{5}$, Elin Larsson ${ }^{6}$, Richard Lundmark ${ }^{6}$, Matthias Kern ${ }^{7}$, Matthias Blüher $^{7}$, Dominik N. Müller ${ }^{8}$,

5 Volker Haucke ${ }^{5}$, Annette Schürmann ${ }^{4}$, Carmen Birchmeier², Oliver Daumke ${ }^{1,9, *}$

6

$7 \quad{ }^{1}$ Crystallography, Max-Delbrück-Center for Molecular Medicine, Berlin, Germany

82 Signal Transduction/Developmental Biology, Max-Delbrück-Center for Molecular Medicine, Berlin, 9 Germany

$10{ }^{3}$ Electron Microscopy Facility, Max-Delbrück-Center for Molecular Medicine, Berlin, Germany

$11{ }^{4}$ Experimental Diabetology, German Institute of Human Nutrition, German Center for Diabetes Research, 12 München-Neuherberg, Potsdam, Germany

$13{ }^{5}$ Dept. of Molecular Pharmacology \& Cell Biology and Imaging Core Facility, Leibniz-Forschungsinstitut für 14 Molekulare Pharmakologie, Berlin, Germany

$15{ }^{6}$ Integrative Medical Biology, Umeå University, 90187 Umeå, Sweden

$16{ }^{7}$ Department of Medicine, University of Leipzig, Leipzig, Germany

$17{ }^{8}$ Experimental \& Clinical Research Center, a cooperation between Charité Universitätsmedizin Berlin and 18 Max Delbrück Center for Molecular Medicine, Berlin, Germany

$19{ }^{9}$ Institute of Chemistry and Biochemistry, Freie Universität Berlin, Takustrasse 6, 14195 Berlin, Germany

$20 \quad{ }^{10}$ These authors contributed equally

21

22

$23 *$ Corresponding authors

24 Correspondence: claudia.matthaeus@mdc-berlin.de; oliver.daumke@mdc-berlin.de 


\section{Abstract}

27 Eps15-homology domain containing protein 2 (EHD2) is a dynamin-related ATPase located at the neck of

28 caveolae, but its physiological function has remained unclear. Here, we found that global genetic ablation

29 of EHD2 in mice led to increased fat accumulation. This organismic phenotype was paralleled at the

30 cellular level by increased lipid uptake via a caveolae-, dynamin- and CD36-dependent pathway, an

31 elevated number of detached caveolae and higher caveolar mobility. Furthermore, EHD2 expression itself

32 was down-regulated in the visceral fat of two obese mouse models and obese patients. Our data suggest

33 that EHD2 controls a cell-autonomous, caveolae-dependent lipid uptake pathway and suggest that low

34 EHD2 expression levels are linked to obesity.

35

36

37 Keywords: EHD2, caveolae, fatty acid uptake, lipid metabolism, obesity 


\section{Introduction}

Caveolae are small membrane invaginations of the plasma membrane that are abundantly found

41 in adipocytes, endothelial and muscle cells (1). They have been implicated in the regulation of membrane

42

43

44

tension (2,3), in mediating lipid metabolism (4), or in acting as distinct sites for specific and highly regulated signaling cascades such as the endothelial nitric oxide synthase (eNOS)-nitric oxide (NO) pathway (5). The characteristically shaped caveolar bulb has a typical diameter of $50-100 \mathrm{~nm}$ and is connected to the cell surface via a narrow neck region. The integral membrane protein Caveolin (with three isoforms in human, Cav1-3) and the peripheral membrane protein Cavin (with four isoforms in human, Cavin1-4) build a mesh-like coat around the caveolar bulb (6-10). In addition, BAR domain containing proteins of the PACSIN/syndapin family (PACSIN1-3 in human) participate in the biogenesis of caveolae (11-13).

Loss of Cav1/Cav3 or Cavin1 results in a complete lack of caveolae from the plasma membrane (4, 14, 15). Cav1 KO mice suffer from cardiomyopathy, pulmonary hypertension, endothelium-dependent relaxation problems and defective lipid metabolism (1). In agreement with the latter, Cav1 KO mice are resistant to high fat diet-induced obesity (16) and display smaller white adipocytes and fat pads (17). Furthermore, increased levels of triglycerides and fatty acids are found in blood plasma samples obtained from Cav1 KO mice suggesting a reduced cellular uptake of fatty acids (16). A similar metabolic phenotype was found in mice lacking Cavin1 $(4,18)$. Conversely, overexpression of Cav1 in adipocytes results in an increased number of caveolae, enhanced fat accumulation, enlarged adipocytes and lipid droplets (LDs) (19). These results suggest that caveolae are involved in lipid accumulation in adipocytes and may promote fatty acid uptake (20). However, the molecular mechanisms of caveolae-dependent fat uptake have remained obscure.

Eps15 homology domain containing protein 2 (EHD2) localizes to the caveolar neck region $(6,21$, 22). The protein belongs to the dynamin-related EHD ATPase family, which comprises four members in 

(23). EHD is built of an N-terminal GTPase (G)-domain, which mediates dimerization and oligomerization, a helical domain containing the membrane binding site, and a C-terminal regulatory Eps15 homology (EH)domain. The proteins exist in a closed auto-inhibited conformation in solution (24). When recruited to membranes, a series of conformational changes aligns the phospholipid binding sites with the membrane and facilitates oligomerization of EHD2 into ring-like structures (25-27). mobility of caveolae, whereas EHD2 overexpression stabilizes caveolae at the plasma membrane $(9,21$,

$7122,28)$. This led to the hypothesis that formation of an EHD2-ring at the neck of caveolae restricts caveolar

72 mobility within the membrane. In agreement with this hypothesis, EHD2 assembles in an ATP-dependent

73 fashion into ring-like oligomers in vitro and induces the formation of tubular liposomes with an inner

74 diameter of $20 \mathrm{~nm}$, corresponding to the diameter of the caveolar neck (24). Whether EHD2 also controls caveolar membrane dynamics in vivo and what the physiological consequences of EHD2 loss at the organismic level are, is unknown. In this study, we found that EHD2 KO mice display enlarged lipid accumulation in white and brown adipose tissue, and increased lipid droplets (LDs) in caveolae-harboring cell types like adipocytes or fibroblasts. In adipose tissue lacking EHD2, caveolae were frequently detached from the plasma membrane and displayed elevated mobility. Furthermore, in two obesity mouse models, as well as in white adipose tissue of obese patients, reduced EHD2 expression and an increased number of detached caveolae were found in visceral fat. Our data establish EHD2 as a negative regulator of caveolae-

84 obesity. 


\section{Results} \\ Generation of EHD2 knockout mice}

To examine the physiological function of EHD2, a mouse strain with LoxP recognition sites surrounding exon 3 and intron 3 of the Ehd 2 gene was engineered (Fig. 1A). Exon 3 encodes part of the highly conserved GTPase domain (residues 137-167), and its deletion is predicted to result in nonfunctional protein. Following global removal of exon 3 by crossings with a germ-line specific Cre-deleter strain, offspring mice were back-crossed with the C57BL6/N mouse strain for five generations, yielding a global EHD2 KO mouse model. Genotyping of offspring confirmed the successful deletion of EHD2 exon 3 in EHD2 del/del animals (Fig. S1A) and real-time PCR revealed the absence of EHD2 mRNA in the EHD2 del/del tissue (Fig. 1B). Western blot analysis indicated the complete loss of EHD2 in WAT and various other tissues of EHD2 del/del mice compared to abundant EHD2 expression in EHD2 +/+ mice (Fig. 1C, Fig. abolished (Fig. 1D).

\section{Loss of EHD2 results in increased lipid accumulation}

EHD2 del/del mice were born in normal Mendelian ratios, were fertile and did not show any obvious phenotype upon initial inspection. In contrast to the reported loss of white fat mass in Cav1 and

107 lipodystrophic and did not display any detectable weight difference when compared to EHD2 del/+ mice

108 (Fig. 1E). However, analysis of the lipid composition in WAT indicated an increased amount of storage 
111 increased deposits of epigonadal and periinguinal white fat (Fig. 1G) indicating that the storage capacity

112 of normal adipose tissue was dysregulated.

When analyzed at the cellular level, white adipocytes of EHD2 del/del mice showed an increased cell size compared to adipocytes from EHD2 del/+ mice or adult C57BL6/N mice (Fig. 1H, Fig. S2A), likely

115 due to increased lipid storage. In contrast to EHD2 del/+ BAT with its distinct brown appearance, EHD2 del/del BAT showed increased beige and white coloring (Fig. 1I). Histological inspections of BAT paraffin

117 and cryostat sections stained against the LD coat protein Perilipin1 indicated an increased LD size in EHD2 del/del BAT compared to EHD2 del/+ or C57BL6/N mice (Fig. 1J, Fig. S2B-C). As no significant differences lipid accumulation (Fig. S2) were found in EHD2 del/+ and C57BL6/N male mice and to reduce animal numbers, the following experiments were carried out with EHD2 del/+ as control group to EHD2 del/del

121 male mice. investigated if adipocyte differentiation is altered in EHD2 del/del WAT. However, adipogenic marker genes like PPARy, Retn or Serpina3k displayed no significant expression change in EHD2 del/del WAT compared to EHD2 del/+ WAT (Fig. S2D). The weight adaptation of both genotypes suggested the occurrence of compensatory mechanisms. First, we looked for changes of adiponectin, leptin and insulin plasma levels but did not detect any significant difference in mice lacking EHD2 compared to EHD2 del/+ mice (Fig. S3). Free fatty acid concentration in blood plasma was slightly reduced in EHD2 del/del suggesting a possible increase in fatty acid uptake (Fig. S3). Furthermore, we observed a down-regulation mechanism that partially compensates for the increased lipid accumulation. 


\section{Increased lipid droplet size in adipocytes lacking EHD2}

To characterize the mechanisms of increased lipid accumulation in EHD2 del/del fat cells, lipid metabolism was investigated in cultured adipocytes. Primary pre-adipocytes were isolated from WAT of EHD2 del/+ and EHD2 del/del mice and differentiated into mature adipocytes, followed by BODIPY staining to measure LD sizes. Undifferentiated EHD2 del/del pre-adipocytes showed increased LD size, a difference that was even more pronounced in differentiated EHD2 del/del adipocytes compared to EHD2 del/+ (Fig. 2A-B). 3D reconstruction of EHD2 del/del differentiated adipocytes illustrates an extensively increased volume of some LDs (Fig. 2C).

We addressed the possibility that increased lipid uptake is a secondary effect of EHD2 deletion mediated via putative organ cross-talk. We therefore repeated the experiments with cultivated adipocytes derived from EHD2 cKO flox/flox mice, in which EHD2 expression was down-regulated by expression of Cre recombinase via viral transfection (AAV8). Again, EHD2 removal led to increased LD growth (Fig. 2D-E), indicating a cell-autonomous function of EHD2 in controlling lipid uptake.

LD growth is mainly mediated by extracellular fatty acid uptake and conversion into triglycerides, whereas increased glucose uptake and de novo lipogenesis play minor roles in this process $(29,30)$. Fatty acids and lipids are present at high concentrations in fetal bovine serum (FBS). Addition of delipidated FBS during adipocyte differentiation resulted in complete loss of LD in both genotypes (Fig. S4A), whereas glucose-depletion led to a general impairment of adipocyte differentiation. Importantly, enlarged LDs were still observed in KO adipocytes even under conditions of glucose depletion (Fig. S4A). These data led us to hypothesize that the increased LD size in EHD2 del/del adipocytes may be a consequence of increased fatty acid uptake, in line with the suggested function of caveolae in fatty acid uptake (31-33).

To test this possibility directly, we monitored the uptake of extracellularly added fatty acids into differentiated adipocytes using BODIPY-labelled dodecanoic acid (FA12) paired with FACS analysis. After 5 min, only a minor fraction of EHD2 del/+ adipocytes displayed intense BODIPY staining (R2, for definition 
see Fig. 2F). This R2 population increased to more than $30 \%$ after 60 min of FA12 treatment. EHD2 del/del adipocytes displayed increased BODIPY staining at both early and late time points (Fig. 2F-H; Fig. S4B-D), indicating accelerated lipid uptake. This conclusion was further supported by light microscopy imaging, which revealed more intense BODIPY staining of EHD2 del/del cells compared to EHD2 del/+ adipocytes after 60 min of fatty acid incubation (Fig. 2G). In contrast, EHD2 del/+ and EHD2 del/del adipocytes did not differ with respect to their ability to take up extracellularly added glucose (Fig. S4E-G). Previously, an involvement of EHD2 in the autophagic engulfment of LDs (lipophagy) was suggested (34). However, inducing starvation by incubation of differentiated adipocytes with Hank's balanced salt solution (HBSS) revealed no differences in the release of stored lipids in EHD2 del/+ and EHD2 del/del adipocytes, and both genotypes displayed similar reductions in lipid accumulation (Fig. S5A). These data indicate that loss of EHD2 does not affect the release of fatty acids or lipophagy, but specifically controls LD size by regulating fatty acid uptake.

\section{Loss of EHD2 results in detachment of caveolae from the plasma membrane in vivo}

Given the known role of caveolae in the uptake of fatty acids (16), we hypothesized that EHD2 may restrict fatty acid uptake by controlling caveolar function and/ or dynamics. To test whether loss of EHD2 affects caveolar morphology, WAT and BAT were analyzed by electron microscopy (EM). Caveolae in EHD2 del/+ BAT were mostly membrane-bound and displayed the characteristic flask-shaped morphology (Fig. 3A, white arrows, ratio detached/membrane bound caveolae $=0.27$ ). Strikingly, an increased number of caveolae appeared detached from the plasma membrane in BAT isolated from EHD2 del/del mice compared to EHD2 del/+ controls, as judged from the complete closure of the lipid bilayer in the plasma membrane and the caveolae (Fig. 3A, B, black arrows, ratio detached/membrane bound caveolae $=1.75)$. The total number of caveolae, as well as the caveolar diameter and size, were unchanged in brown adipocytes lacking EHD2. 
An increased number of detached caveolae were also observed in EHD2 del/del white adipocytes compared to EHD2 del/+ cells from littermate controls (Fig. 3C, D, black and white arrow heads, ratio detached $/$ membrane bound caveolae $(\mathrm{del} / \mathrm{del})=1.2 \mathrm{vs}$. ratio $(\mathrm{del} /+)=0.24)$. In white adipocytes, the total number of caveolae was reduced, while both caveolar size and diameter were increased in EHD2 del/del compared to EHD2 del/+ animals (Fig. 3D). Cav1 immunogold labeling confirmed that the round vesicles close the plasma membrane, indeed, were detached caveolae (Fig. 3E). 3D visualization of EHD2 del/del 2D EM images were not connected to the plasma membrane (Fig. 3F, G, Movie S1) but localized 20-30 nm underneath (Fig. 3G a, b), although some caveolae close to the plasma membrane showed thin of caveolae from the plasma membrane in EHD2 del/del adipocytes, suggesting a crucial function for EHD2 in the stabilization of caveolae at the plasma membrane.

\section{Increased caveolar mobility in EHD2 knockout cells}

To further dissect the interplay of caveolar mobility and LD growth at the molecular level, we investigated caveolar mobility and endocytosis in mouse embryonic fibroblasts (MEFs) by total internal reflection fluorescence (TIRF) microscopy. MEFs derived from EHD2 +/+ and del/del mice were transfected with pCav1-EGFP to label single caveolae. As illustrated in Fig. 4A, regions of moderate Cav1 expression were investigated to ensure that distinct Cav1 spots were observed during the analysis. Live TIRF imaging 
207 significantly decreased following depletion of Cav1 (Fig. 5D, E), indicating that the effects of EHD2 on LD

fixed, non-moving Cav1 spots (referred to as lines in Fig. 4B) in EHD2 del/del MEFs compared to EHD2 +/+ cells. Moreover, a larger number of highly mobile Cav1 sparks, reflecting fast moving caveolae, was found in EHD2 del/del cells (Fig. 4A, B). Re-expression of EHD2 in EHD2 del/del MEFs reduced the mobility of caveolae, often leading to their immobilization (Fig. S6, Movie S4).

\section{Determinants of EHD2-mediated fatty acid uptake}

We further characterized the determinants of the observed lipid uptake in MEFs. Similar to EHD2 del/del adipocytes, EHD2 del/del MEFs showed increased lipid accumulation and LD size after adipogenic differentiation, as illustrated by Oil red $O$ staining (Fig. S7A) and BODIPY staining (Fig. S7B, C). Furthermore, both storage and membrane lipids were increased in MEFs lacking EHD2 (Fig. S7D). Reexpression of an EGFP-tagged EHD2 version in in EHD2 +/+ and del/del MEFs rescued the observed LD phenotype, even reducing the size of LDs compared to EGFP expressing cells (Fig. 5A, B). These data indicate a general and cell autonomous role of EHD2 in the control of LD growth and size that is not restricted to fat cells.

To identify the functional regions within EHD2 that are crucial for regulating lipid uptake, we transfected EHD2 del/del MEFs with various EGFP-tagged EHD2 deletion constructs. EHD2 constructs lacking the N-terminus, the EH-domain or both, rescued EHD2 loss, resulting in smaller LDs (Fig. 5C, S7E). In contrast, expression of single EHD2 mutants affecting membrane binding (F322A) or oligomerization/ATPase activity of EHD2 (F122A) did not reduce LD size, indicating a crucial role of these properties for EHD2 function.

To analyze if the observed phenotype in EHD2 del/del MEFs was dependent on caveolae, MEFs lacking EHD2 were treated with Cav1 siRNA to eliminate caveolae. LD size in EHD2 del/del MEFs was size require and likely are mediated by caveolae. 
It was previously reported that dynamin is located on caveolae (35). Consistent with a role of dynamin in caveolae function, overexpression of a dynamin 2 dominant negative mutant (pGFP-Dyn2K44A, Fig. 5F, G) completely abolished the size increase of LDs in EHD2 del/del MEFs, suggesting a role for dynamin in fatty acid uptake via caveolae.

Previous work has implicated the fatty acid binding membrane protein CD36 in caveolaedependent fatty acid uptake $(31,36,37)$. To probe a possible function of CD36 in EHD2-dependent lipid uptake, CD36 expression in MEFs was downregulated by treatment with either one of three specific CD36 siRNA. Antibody staining confirmed the efficient knockdown of CD36 in EHD2 +/+ and del/del MEFs (Fig. 5H). Removal of CD36 in EHD2 +/+ and del/del MEFs dramatically decreased the size of LDs compared to a control siRNA treated cells (Fig. 5H). Hence, the observed enlargement of LDs in cells lacking EHD2 depends on CD36. These converging lines of evidence suggest that caveolae dynamics is key to the regulation of fatty acid internalization and that this is coupled to the previously shown importance of CD36 and caveolin as fatty acid binding proteins.

\section{Decreased EHD2 expression in genetic obesity models or in diet induced obesity}

Our data indicate an EHD2-dependent regulation of lipid uptake in adipose tissue. If this were of physiological relevance, one might expect EHD2 expression to be dysregulated in obese mice and men. We therefore investigated if EHD2 expression is altered in two obesity-related mouse models, ob/ob and NZO (38). Indeed, WAT obtained from ob/ob and NZO mice showed reduced EHD2 expression compared to C57BL6/N mice fed by standard diet (Fig. 6A). When investigating adipocytes from ob/ob mice, a higher proportion of detached caveolae were found in the obesity mouse model (ratio detached/membrane bound caveolae $=1.4$ vs. 0.35 in C57BL6/N mice fed with standard diet, Fig. 6B, C). In addition, we analyzed EHD2 expression in visceral and subcutaneous WAT from patients ranking in their body mass index (BMI) from normal to morbid obesity (BMI<25 - BMI >40, Fig. 6D, E). In both depots, EHD2 expression was 
highest in normal weight subjects and was significantly lower in overweight and obese people, whereas

256 EHD2 expression did not differ between different obesity stages. These data imply that EHD2 expression

257 is regulated by lipid uptake and load and suggest that EHD2-mediated caveolar dynamics may be altered

258 in obesity.

\section{Discussion}

Here, we identify EHD2 as a negative regulator of caveolae-dependent lipid uptake. Loss of EHD2 resulted in increased lipid accumulation, which was observed in adipose tissue of the whole organism as well as in cell culture-based experiments. Loss of EHD2 was associated with the detachment of caveolae

264 from the plasma membrane, higher caveolar mobility and increased lipid uptake. We demonstrate that caveolae, dynamin 2 and the fatty acid translocase CD36 play a role in the EHD2-dependent lipid uptake pathway. In addition, obese mouse models exhibit decreased EHD2 expression in WAT and in addition,

267 also obese patients showed a reduction in EHD2 gene expression. Thus, our study reveals a cellautonomous caveolae dependent lipid uptake route that is controlled by EHD2 and modified by metabolic conditions.

271 reduced fat mass and did not develop any form of obesity. In addition, Pohl et al. (39) observed decreased

272 oleate uptake after expression of a dominant-negative Cav1 mutant. The EHD2 KO mouse model, 273 described here, revealed the opposite phenotype, e.g. a caveolae gain-of-function in vivo model. In EHD2

274 KO mice, caveolae were more often detached from the plasma membrane and showed a higher mobility 275 and faster lipid uptake, resulting in enlarged LDs. Unlike in Cav1 over-expressing mice (19), which also 276 show increased fatty acid uptake, the number of caveolae was not increased in EHD2 KO mice. This 277 supports a model in which not only caveolae number, but also caveolar dynamics play a crucial role in this 278 process (Fig. 6F). Furthermore, such idea is in line with our structural findings that EHD2 can form ring- 
like oligomers that may stabilize the neck of caveolae (24-27), thereby restricting caveolar mobility (21, 22). Based on studies in EHD2 KO NIH $3 T 3$ cell line, Yeow et al. (40) suggested that EHD1 and/or EHD4 could rescue loss of EHD2 during its role in membrane protection. However, we did not find rescue of caveolae detachment and lipid uptake in EHD2 KO cells by other EHD family members.

The detailed pathway of caveolae-dependent cellular lipid uptake has been intensively studied (4, $20,31,32,41)$, but the exact molecular mechanisms are still unclear. Our observation indicate that fatty acid uptake depends on caveolar dynamics, detachment and most likely caveolae endocytosis and is regulated by EHD2. However, it remains elusive how caveolar shuttling is linked to the growth of lipid droplets. The EHD2 knockout model may be a useful tool to further dissect the molecular lipid uptake mechanism.

The loss of EHD2 on the cellular level led to increased fat deposits on the organismic level, which was particularly evident in older animals. The observed phenotype based on the global loss of EHD2 could be influenced by organ-organ interactions (42). However, we did not find any evidence for differences in adipocyte derived secretory factors, such as leptin (Fig. S3). Furthermore, increased lipid uptake was dependent on caveolae, as shown by Cav1 knockdown experiments, and lipid droplet growth could specifically be induced by viral transfection of Cre recombinase in EHD2 cKO flox/flox, but not in flox/wt adipocytes. Thus, increased lipid uptake is caused by a cell-autonomous, caveolae-dependent mechanism. As the WAT distribution and lipid accumulation is known to differ in male and female mice, further studies are required to analyze potential sex-specific differences in EHD2-dependent lipid uptake.

Despite the observed lipid accumulation in mice lacking EHD2, the body weight of EHD2 del/del and EHD2 del/+ mice was unaltered, suggesting metabolic compensation. In line with this idea, EHD2 del/del WAT and cultivated EHD2 del/del adipocytes showed a significant reduction in expression levels of genes involved in de novo lipogenesis like SREBP1 or FAS indicating a strong downregulation of glucosedependent fatty acid production in fat cells. Similar compensatory mechanisms were noted in patients 
uptake and consequently help to treat obesity in patients.

In conclusion, our study reveals that EHD2 controls a caveolae-dependent cellular lipid uptake

312 pathway.

\section{Methods}

315 Please see SI for detailed description of all methods and material.

EHD2 delta E3 mouse strain generation. The EHD2 targeting construct was generated by insertion of two lox $\mathrm{P}$ sequences flanking exon 3 of EHD2 genomic DNA by homologous recombination in E.coli as previously described (47) including a pGK Neomycin and a diphtheria toxin A (DTA) cassette. Electroporation of the linearized targeting vector in R1 ES cells was performed. Mice carrying a loxPflanked Exon 3 of EHD2 gene were mated to Cre deleter mice to generate EHD2 mutant (del/del) mice.

321 After backcrossing the EHD2 del/del mice with C57BL6/N (Charles River, between 20-30 weeks, male) for

3226 generations only male EHD2 del/del or EHD2 del/+ (as control) mice were used and littermates were

323 randomly assigned to experimental groups. All animals were handled accordingly to governmental animal 324 welfare guidelines and were housed under standard conditions.

325 Obesity Mouse Models. Male NZO/HIBomDife (German Institute of Human Nutrition, Nuthetal, 326 Germany), C57BL/6J (Charles River Laboratories, Sulzfeld, Germany) and B6.V-Lepob/ob/JBomTac (B6327 ob/ob) mice (Charles River Laboratories, Calco, Italy) were housed under standard conditions 328 (conventional germ status, $22{ }^{\circ} \mathrm{C}$ with 12 hour /dark cycling). NZO and C57BL/6J mice were fed were fed 329 standard chow diet (Ssniff, Soest). Starting at 5 weeks of age B6-ob/ob received carbohydrate free diet 330 (48). Mice were sacrificed at an age of 20-22 weeks. 
Oil Red $\mathrm{O}$ staining. LDs in tissue sections or cultivated adipocytes and MEFs were stained with Oil Red O as published by (49).

Human EHD2 expression. All participants were recruited by University Leipzig (approval numbers: 26508, 159-12-21052012, and 017-12-23012012) and samples were treated as previously described (50). (Sigma) coated glass dishes (ThermoFisher). Cells were washed with PBS, treated with 4\% PFA for 10 min and blocking buffer (1\%donkey serum/1\% TritonX100/PBS) for 20 min. The first antibody was incubated for $1 \mathrm{~h}$, followed by secondary antibody and DAPI stain. For LD staining, BODIPY (Invitrogen, saturated solution) or Nile Red (Sigma, saturated solution) was diluted to 1:1000 in PBS and applied for $30 \mathrm{~min}$. The stained cells were washed and the glass dishes were placed on conventional microscope slides and embedded in ImmoMount. Zeiss LSM700 or Zeiss LSM880 microscopes and ImageJ/Fij were used.

Transmission Electron microscopy (TEM). Mice were fixed by perfusion with $4 \%(\mathrm{w} / \mathrm{v})$ formaldehyde in $0.1 \mathrm{M}$ phosphate buffer. Tissue blocs were postfixed in phosphate buffered $2.5 \%(\mathrm{v} / \mathrm{v})$ glutaraldehyde, treated with $1 \%(\mathrm{v} / \mathrm{v})$ osmium tetroxide, dehydrated in a graded series of ethanol and embedded in the PolyBed $^{\circledR} 812$ resin (Polysciences Europe $\mathrm{GmbH}$ ). Ultrathin sections $(60-80 \mathrm{~nm}$ ) were cut (Leica microsystems) and stained with uranyl acetate and lead citrate before image acquisition. Samples were examined at $80 \mathrm{kV}$ with a Zeiss EM 910 electron microscope. Acquisition was done with a Quemesa CDD camera and the iTEM software (Emsis $\mathrm{GmbH})$.

Electron tomography (ET). To obtain electron tomograms $250 \mathrm{~nm}$ slices of EHD2 del/del BAT were prepared of samples embedded in resin and treated as described for TEM. The samples were tilted from 60 to $-60^{\circ}$ in $2^{\circ}$ steps and examined at $120 \mathrm{kV}$ with a FEI Talos electron microscope. FEl tomography software was used for acquisition of tomograms, detailed analysis and reconstruction was done with Inspect3D, Amira (both obtained from FEI) and IMOD (University of Colorado, USA).

354 Fatty acid uptake assay. EHD2 del/+ and EHD2 del/del pre-adipocytes were seeded in 6-well plates (100.000 cells/well) and differentiated in mature adipocytes as described above. The fatty acid uptake assay was performed as described elsewhere (51). Briefly, differentiated adipocytes were starved for $1 \mathrm{~h}$ with serum-free DMEM. Next, $2 \mu \mathrm{M}$ dodecanoic acid (FA12) labelled with BODIPY (Molecular probes \#D3822) diluted in serum-free DMEM + $10 \mu \mathrm{g} / \mathrm{ml}$ insulin was added to the adipocytes and incubated for 5-60 min at $37^{\circ} \mathrm{C}$ followed by FACS. Glucose uptake was measured with $200 \mu \mathrm{M}$ 2-NBDG (2-deoxy-2-[(7nitro-2,1,3-benzoxadiazol-4-yl)amino]-D-glucose, molecular probes \#N13195) diluted in serum-free 


\section{Acknowledgments}

364

365

366

367

368

369

370

371

372

373

374

375

376

377

378

379

380

381

382

We thank Petra Stallerow for taking care of the EHD2 delta E3 mouse strain, Karin Jacobi for advice with the animal application, Vivian Schulz and Carola Bernert for helping with the genotyping of the mice, Claudio Shah for purifying the EHD2 antiserum, and the advanced light imaging facility at MDC for technical support. The authors acknowledge financial support from the Deutsche Forschungsgemeinschaft (DFG; SFB 958/A7 to V.H., A12 to O.D., A13 to A.S.) and support by The Initiative and Networking Fund of the Helmholtz Association.

\section{Author Contributions}

C.M. planned, performed and analyzed all experiments if not otherwise indicated. C.M. and O.D. wrote the manuscript, with input from all authors. S.K. performed and analyzed all EM imaging, C.M. analyzed EM images, S.K. and C.M. performed and analyzed ET. I.L. generated the EHD2 KO mouse model and performed in situ hybridization. W.J. analyzed blood plasma markers and EHD2 expression in obesity mouse models. M.L. helped during TIRF imaging and discussed experiments, A.M. isolated primary MEF and performed the EHD2 Western Blot. E.L. performed lipid droplet staining experiments after Cav1 knockdown. M.K. and M.B. recruited obese patients and handled human samples. A.S., V.H., R.L., C.B. and D.N.M. discussed potential experiments and the manuscript. O.D. wrote the mouse animal application with help of D.N.M. and C.M.

\section{Declaration of Interests}

The authors declare no competing interests.

\section{References}

1. Cheng JPX, Nichols BJ (2016) Caveolae : One Function or Many ? Trends Cell Biol 26(3):177-189.

2. Sinha B, et al. (2011) Cells respond to mechanical stress by rapid disassembly of caveolae. Cell

3. Torrino S, et al. (2018) EHD2 is a mechanotransducer connecting caveolae dynamics with gene transcription. 1-14.

4. Liu L, et al. (2008) Deletion of Cavin/PTRF Causes Global Loss of Caveolae, Dyslipidemia, and Glucose Intolerance. Cell Metab 8(4):310-317.

5. Ju H, Zou R, Venema VJ, Venema RC (1997) Direct Interaction of Endothelial Nitric-oxide Synthase 
and Caveolin-1 Inhibits Synthase Activity. J Biol Chem 272(30):18522-18525.

6. Ludwig A, et al. (2013) Molecular Composition and Ultrastructure of the Caveolar Coat Complex. PLoS Biol 11(8). doi:10.1371/journal.pbio.1001640.

7. $\quad$ Ludwig A, Nichols BJ, Sandin S (2016) Architecture of the caveolar coat complex. J Cell Sci:30773083.

8. Stoeber M, et al. (2016) Model for the architecture of caveolae based on a flexible, net-like assembly of Cavin1 and Caveolin discs. Proc Natl Acad Sci U S A:201616838.

9. Mohan J, Moren B, Larsson E, Holst MR, Lundmark R (2015) Cavin3 interacts with cavin1 and caveolin1 to increase surface dynamics of caveolae. J Cell Sci 128(5):979-991.

10. Kovtun O, Tillu VA, Ariotti N, Parton RG, Collins BM (2015) Cavin family proteins and the assembly of caveolae. J Cell Sci 128(7):1269-1278.

11. Hansen CG, Howard G, Nichols BJ (2011) Pacsin 2 is recruited to caveolae and functions in caveolar biogenesis. J Cell Sci 124(Pt 16):2777-2785.

12. Senju Y, Itoh Y, Takano K, Hamada S, Suetsugu S (2011) Essential role of PACSIN2/syndapin-II in caveolae membrane sculpting. J Cell Sci 124(12):2032-2040.

13. Seemann E, et al. (2017) Deciphering caveolar functions by syndapin III KO-mediated impairment of caveolar invagination. Elife 6:1-37.

14. Drab M, et al. (2001) Loss of caveolae, vascular dysfunction, and pulmonary defects in caveolin-1 gene-disrupted mice. Science (80- ) 293(5539):2449-2452.

15. Hill MM, et al. (2008) PTRF-Cavin, a Conserved Cytoplasmic Protein Required for Caveola Formation and Function. Cell 132(1):113-124.

16. Razani B, et al. (2002) Caveolin-1-deficient mice are lean, resistant to diet-induced obesity, and show hypertriglyceridemia with adipocyte abnormalities. J Biol Chem 277(10):8635-8647.

17. Martin S, et al. (2012) Caveolin-1 Deficiency Leads to Increased Susceptibility to Cell Death and Fibrosis in White Adipose Tissue: Characterization of a Lipodystrophic Model. PLoS One 7(9):1-9.

18. Ding SY, et al. (2014) Pleiotropic effects of cavin-1 deficiency on lipid metabolism. J Biol Chem 289(12):8473-8483.

19. Briand N, et al. (2014) Caveolin-1 expression and cavin stability regulate caveolae dynamics in

20. Pohl J, et al. (2004) Long-Chain Fatty Acid Uptake into Adipocytes Depends on Lipid Raft Function.

21. Morén B, et al. (2012) EHD2 regulates caveolar dynamics via ATP-driven targeting and oligomerization. Mol Biol Cell 23(7):1316-29.

22. Stoeber M, et al. (2012) Oligomers of the ATPase EHD2 confine caveolae to the plasma

23. Uhlén $\mathrm{M}$, et al. (2015) Tissue-based map of the human proteome. 347(6220). 
25. Shah C, et al. (2014) Structural insights into membrane interaction and caveolar targeting of dynamin-like EHD2. Structure 22(3):409-420.

26. Hoernke M, et al. (2017) EHD2 restrains dynamics of caveolae by an ATP-dependent, membranebound, open conformation. Proc Natl Acad Sci U S A 114(22):E4360-E4369.

27. Melo AA, et al. (2017) Structural insights into the activation mechanism of dynamin-like EHD ATPases. Proc Natl Acad Sci 114(22):5629-5634.

28. Shvets E, Bitsikas V, Howard G, Hansen CG, Nichols BJ (2015) Dynamic caveolae exclude bulk membrane proteins and are required for sorting of excess glycosphingolipids. Nat Commun 6:6867-6883.

29. Rutkowski JM, Stern JH, Scherer PE (2015) The cell biology of fat expansion. J Cell Biol 208(5):501-512.

30. Wilfling F, et al. (2013) Triacylglycerol synthesis enzymes mediate lipid droplet growth by relocalizing from the ER to lipid droplets. Dev Cell 24(4):384-399.

31. Ring A, Le Lay S, Pohl J, Verkade P, Stremmel W (2006) Caveolin-1 is required for fatty acid translocase (FAT/CD36) localization and function at the plasma membrane of mouse embryonic fibroblasts. Biochim Biophys Acta - Mol Cell Biol Lipids 1761(4):416-423.

32. Pohl J (2002) Uptake of long-chain fatty acids in HepG2 cells involves caveolae: analysis of a novel pathway. J Lipid Res 43(9):1390-1399.

33. Pilch PF, Meshulam T, Ding S, Liu L (2011) Caveolae and lipid trafficking in adipocytes. Clin Lipidol 6(1):49-58.

34. Li Z, et al. (2016) A novel Rab10-EHBP1-EHD2 complex essential for the autophagic engulfment of lipid droplets. Sci Adv 2(12):1-16.

35. Oh P, McIntosh DP, Schnitzer JE (1998) Dynamin at the neck of caveolae mediates their budding to form transport vesicles by GTP-driven fission from the plasma membrane of endothelium. $J$ Cell Biol 141(1):101-114.

36. Eyre NS, Cleland LG, Tandon NN, Mayrhofer G (2007) Importance of the carboxyl terminus of FAT/CD36 for plasma membrane localization and function in long-chain fatty acid uptake. $J$ Lipid Res 48(3):528-542.

37. Aboulaich N, Vainonen JP, Stralfors P, Vener A V (2004) Vectorial proteomics reveal targeting, phosphorylation and specific fragmentation of polymerase I and transcript release factor (PTRF) at the surface of caveolae in human adipocytes. Biochem J 383(Pt 2):237-48.

38. Kleinert M, et al. (2018) Animal models of obesity and diabetes mellitus. Nat Rev Endocrinol 14(3):140-162.

39. Pohl J, Axel R, Korkmaz U, Ehehalt R, Stremmel W (2005) FAT/CD36-mediated Long-Chain Fatty Acid Uptake in Adipocytes Requires Plasma Membrane Rafts. Mol Biol Cell 16(1):24-31.

40. Yeow I, et al. (2017) Article EHD Proteins Cooperate to Generate Caveolar Clusters and to Maintain Caveolae during Repeated Article EHD Proteins Cooperate to Generate Caveolar Clusters and to Maintain Caveolae during Repeated Mechanical Stress. Curr Biol 27:1-12.

41. Briand N, et al. (2014) Caveolin-1 expression and cavin stability regulate caveolae dynamics in adipocyte lipid store fluctuation. Diabetes 63(12):4032-4044. 
473 42. Stern JH, Rutkowski JM, Scherer PE (2016) Review Adiponectin, Leptin, and Fatty Acids in the Maintenance of Metabolic Homeostasis through Adipose Tissue Crosstalk. Cell Metab 23(5):770784.

43. Guiu-Jurado E, et al. (2015) Downregulation of de novo fatty acid synthesis in subcutaneous adipose tissue of moderately obesewomen. Int J Mol Sci 16(12):29911-29922.

44. Eissing L, et al. (2013) De novo lipogenesis in human fat and liver is linked to ChREBP- $\beta$ and metabolic health. Nat Commun 4:1528-1539.

45. Solinas G, Borén J, Dulloo AG (2015) De novo lipogenesis in metabolic homeostasis: More friend than foe? Mol Metab 4(5):367-377.

482 46. Sonne SB, et al. (2017) Obesity is associated with depot-specific alterations in adipocyte DNA methylation and gene expression. Adipocyte (May):1-10.

47. Liu P, et al. (2003) A Highly Efficient Recombineering-Based Method for Generating Conditional Knockout Mutations. Genome Res 13:476-484.

48. Kluth O, et al. (2015) Identification of Four Mouse Diabetes Candidate Genes Altering $\beta$-Cell Proliferation. PLOS Genet 11(9):e1005506.

49. Mehlem A, Hagberg CE, Muhl L, Eriksson U, Falkevall A (2013) Imaging of neutral lipids by oil red $O$ for analyzing the metabolic status in health and disease. Nat Protoc 8(6):1149-1154.

50. Guiu-jurado E, et al. (2016) Bone Morphogenetic Protein 2 ( BMP2 ) may Contribute to Partition of Energy Storage into Visceral and Subcutaneous Fat Depots. Obesity 24(10):2092-2100.

492 

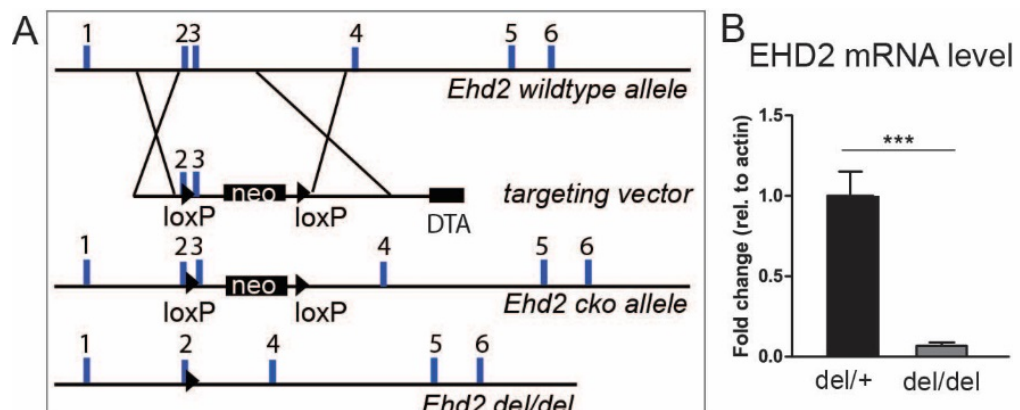

C
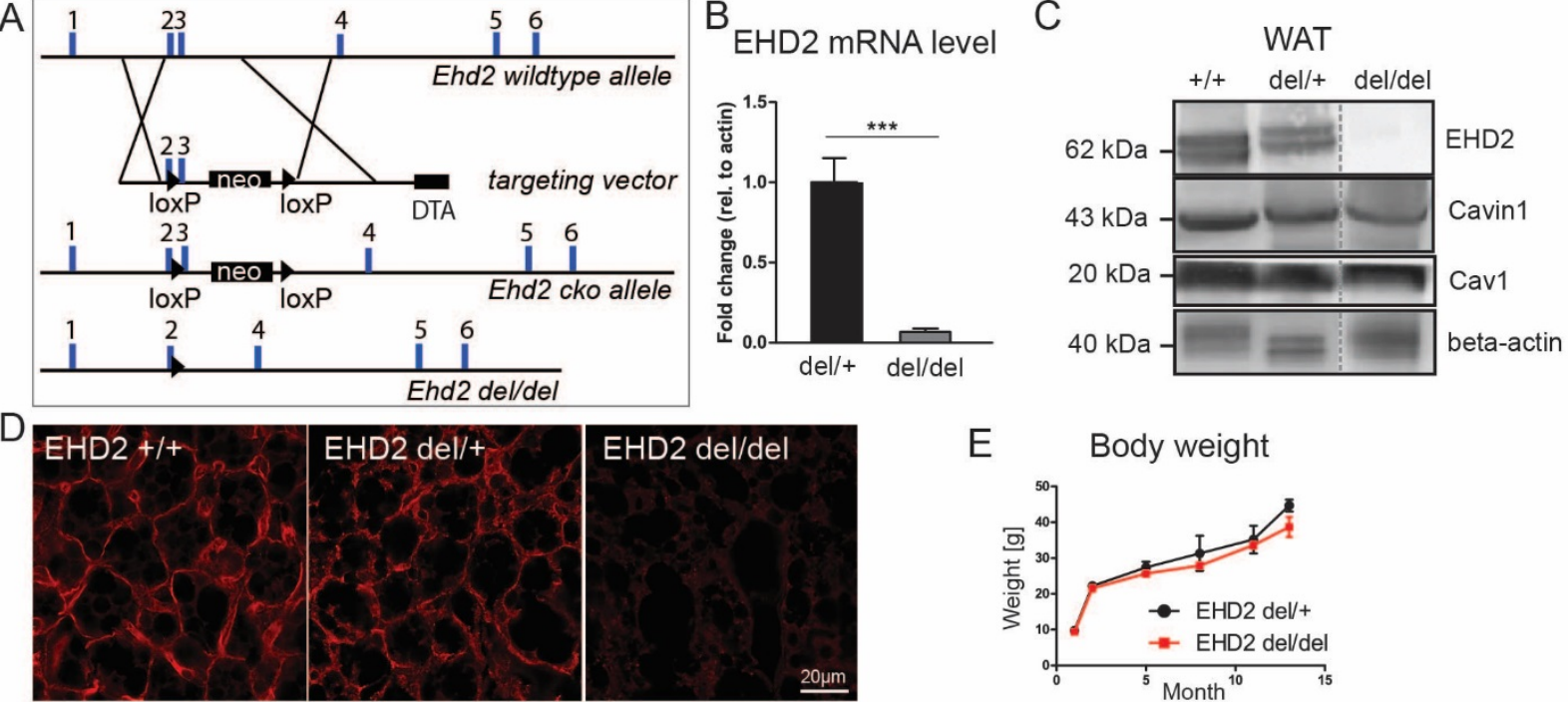

$\mathrm{E}$

Body weight

$\mathrm{F}$

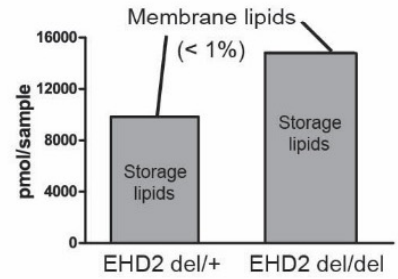

G

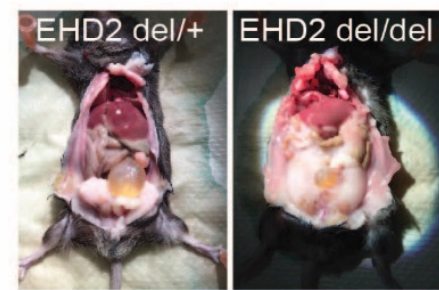

$\mathrm{H}$

White adipocytes
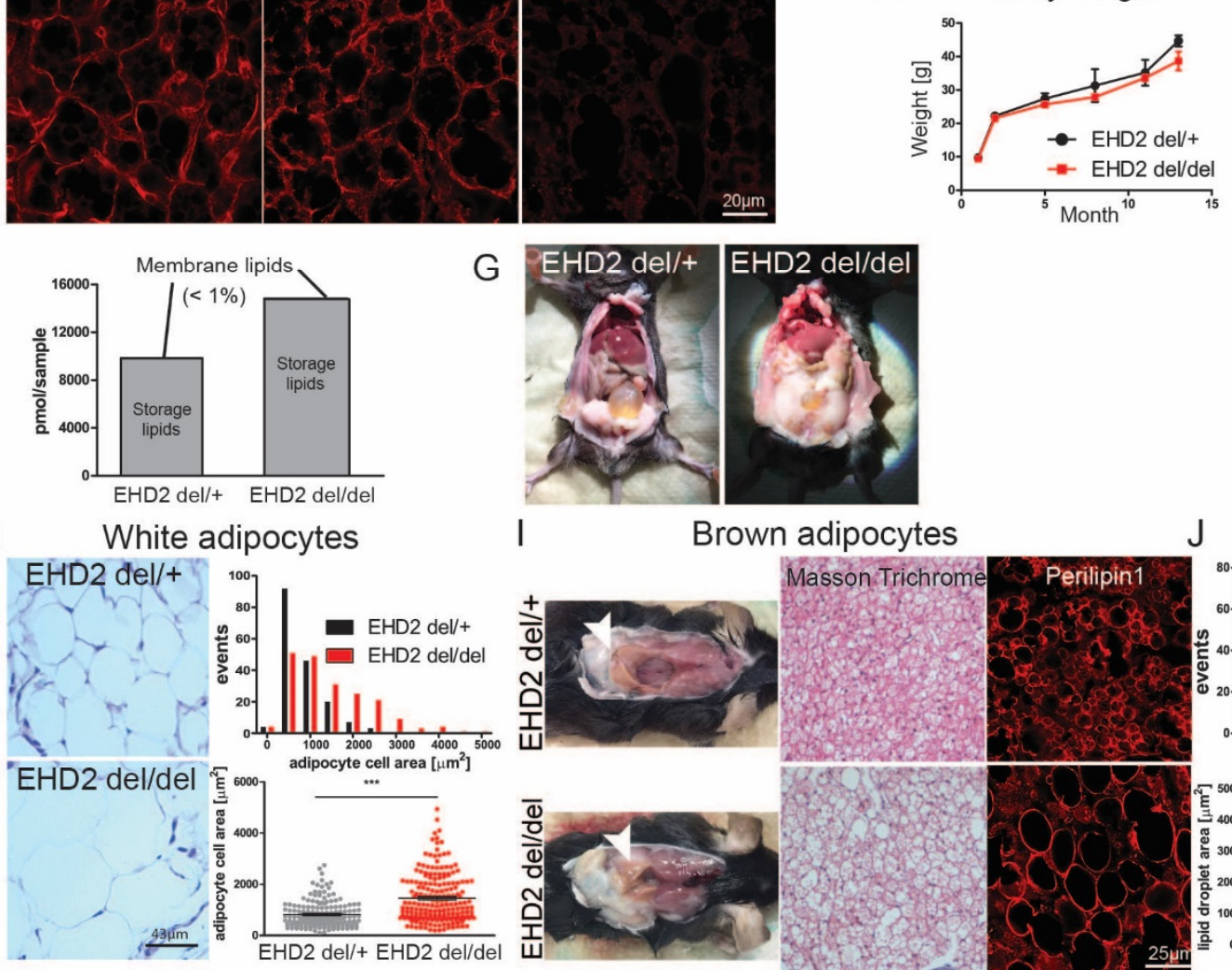

Fig.1 Loss of EHD2 results in increased lipid accumulation in white and brown adipose tissue

A Generation of the EHD2 KO mouse model. A targeting vector containing a pGK-Neomycin (neo) cassette and loxP sites flanking exon 3 was placed in the EHD2 wt allele. EHD2 del/del mice were obtained by breeding with Cre-deleter mouse strain (DTA - diphtheria toxin A).

501 B EHD2 mRNA level in EHD2 del/+ and EHD2 del/del mice (mRNA from BAT, $n=5$ ).

502 C Western Blot analysis of WAT of EHD2+/+, +/- and -/- mice against EHD2, Cav1 and Cavin1.

503 D EHD2 immuno-staining in BAT cryostat sections from EHD2 +/+, del/+ and del/del mice.

504 E The body weight was monitored over 12 months $(n=7)$.

505 F Lipid composition analysis of $15 \mu \mathrm{g}$ WAT obtained from EHD2 del/+ or EHD2 del/del mice.

506 G EHD2 del/+ and EHD2 del/del mice during preparation. 
507 H Masson Trichrome staining of WAT paraffin sections of EHD2 del/+ and EHD2 del/del. Detailed analysis

508 of the adipocytes cell size $(K, n($ del $/+)=172 / 3, n($ del $/$ del $)=199 / 3)$.

509 I EHD2 del/del mice showed decreased BAT in the neck region. Instead, WAT was integrated into the BAT 510 depots.

511 J Masson Trichrome staining of EHD2 del/del BAT paraffin sections and BAT cryostat sections stained

512 against the LD coat protein Perilipin1. LD size was measured in BAT cryostat sections $(M, n(d e l /+)=118 / 3$,

$513 \mathrm{n}($ del $/$ del $)=104 / 3)$.

514 Line graph represents mean +/- SE, column bar graphs show mean + SE, normal distributed groups were

515 analyzed by $\mathrm{t}$-test, not normally distributed values with Mann Withney $\mathrm{U}$ test, $* \mathrm{P}<0.05, * * * \mathrm{P}<0.0001$.

516 For comparison to C57BL6/N, see also Fig. S1-S3.

517

518 
bioRxiv preprint doi: https://doi.org/10.1101/511709; this version posted March 7, 2019. The copyright holder for this preprint (which was no certified by peer review) is the author/funder, who has granted bioRxiv a license to display the preprint in perpetuity. It is made available under aCC-BY-NC-ND 4.0 International license.
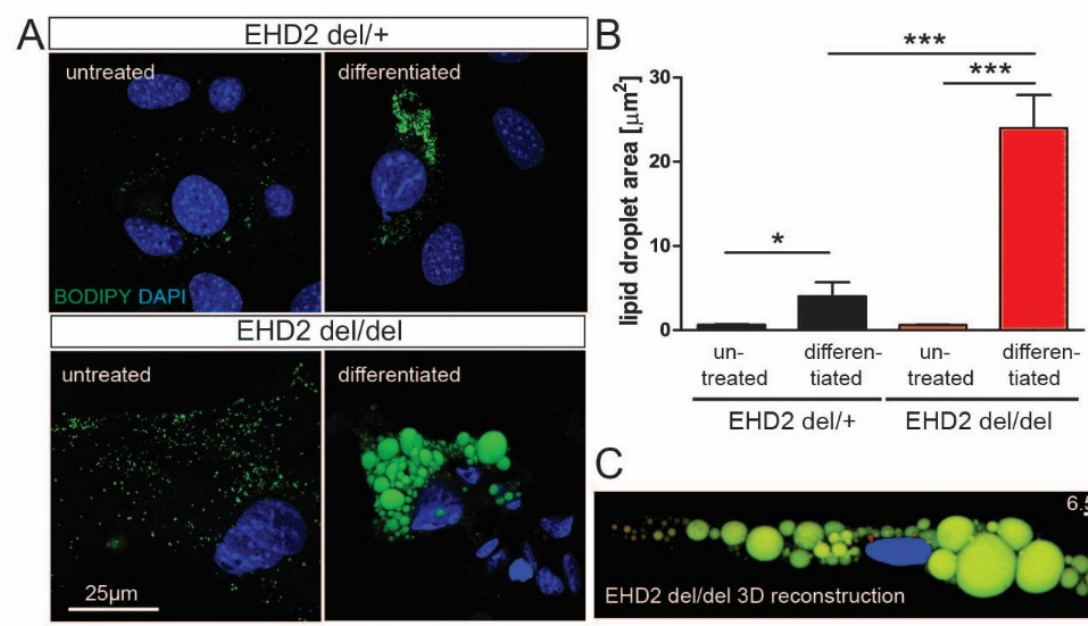

C
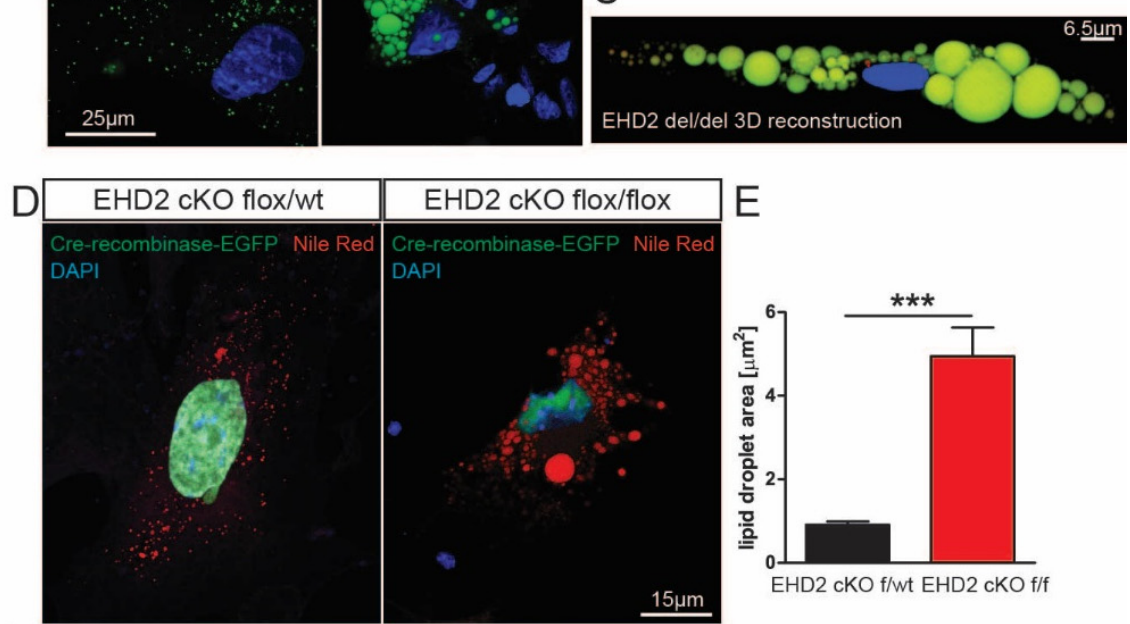

E

$\mathrm{F}$
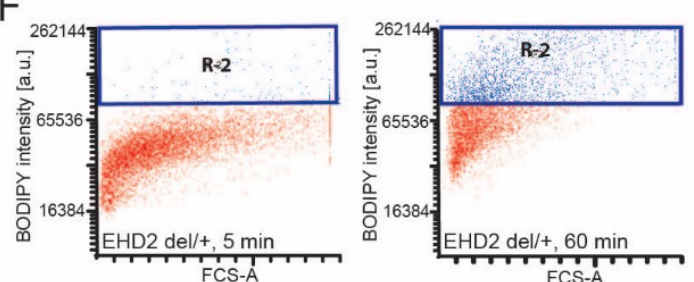

G FA-BODIPY
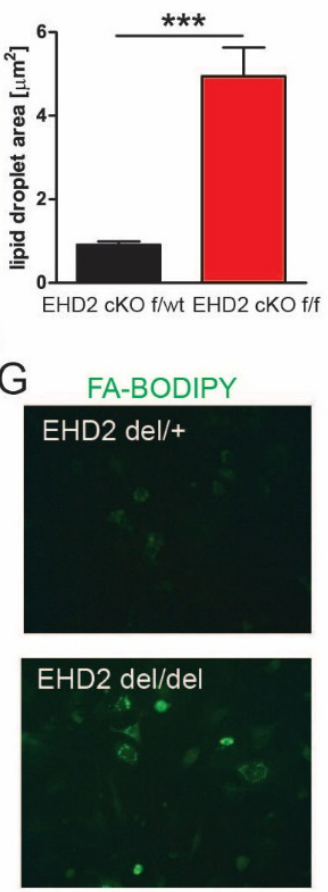

after 60 min incubation

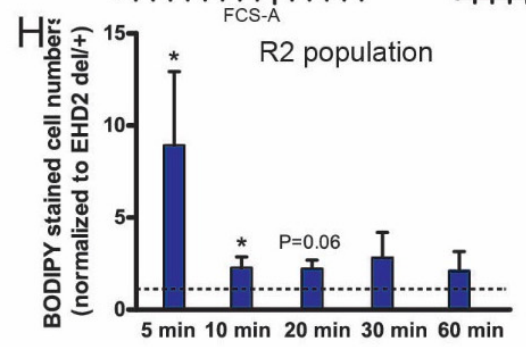


521 A-C Analysis of LD size in EHD2 del/del and EHD2 del/+ adipocytes by staining with BODIPY (untreated:

$522 \mathrm{n}($ del $(+)=74 / 3, \mathrm{n}($ del $/$ del $)=60 / 3)$; differentiated: $\mathrm{n}(\mathrm{del} /+)=132 / 3, \mathrm{n}(\mathrm{del} / \mathrm{del})=95 / 3)$. 3D reconstruction

523 of EHD2 del/del differentiated adipocyte (C). Green - LDs, blue - nucleus.

524 D-E Cultivated EHD2 cKO flox/wt or flox/flox adipocytes were transfected with Cre recombinase-EGFP to

525 induce EHD2 deletion and differentiated for 5 days, lipid droplets were stained with Nile Red for analyzing

526 (n(flox/wt) $=74 / 2, \mathrm{n}($ flox $/$ flox $)=82 / 2$ ).

527 F-H Fatty acid uptake assay in differentiated EHD2 del/+ and EHD2 del/del adipocytes. Dodecanoic acid-

528 BODIPY uptake was measured after 5, 10, 20, 30 or $60 \mathrm{~min}$, and R1 population indicates positively stained

529 cells (illustrated in red in graph F). R2 populations (blue) correspond to higher BODIPY staining intensity

530 in cells and represent adipocytes with increased amount of dodecanoic acid taken up (shown in blue in

531 graphs $F, H)$. Normalization of EHD2 del/del R2 population relative to EHD2 del/+ R2 $(H, n(d e l /+)=6 / 3$

532 experiments, $\mathrm{n}(\mathrm{del} / \mathrm{del})=8 / 3$ experiments). Example images of differentiated adipocytes treated with

533 dodecanoic acid for $60 \mathrm{~min}$ (G, scale bar $40 \mu \mathrm{m}$ ).

534 Column bar graphs illustrate mean +/- SE, t-test or Mann Withney $U$ test were used to calculate significance, * $\mathrm{P}<0.05 ;{ }^{* * *} \mathrm{P}<0.0001$. See also Fig. S4 and S5. 
bioRxiv preprint doi: https://doi.org/10.1101/511709; this version posted March 7, 2019. The copyright holder for this preprint (which was not certified by peer review) is the author/funder, who has granted bioRxiv a license to display the preprint in perpetuity. It is made available under aCC-BY-NC-ND 4.0 International license.
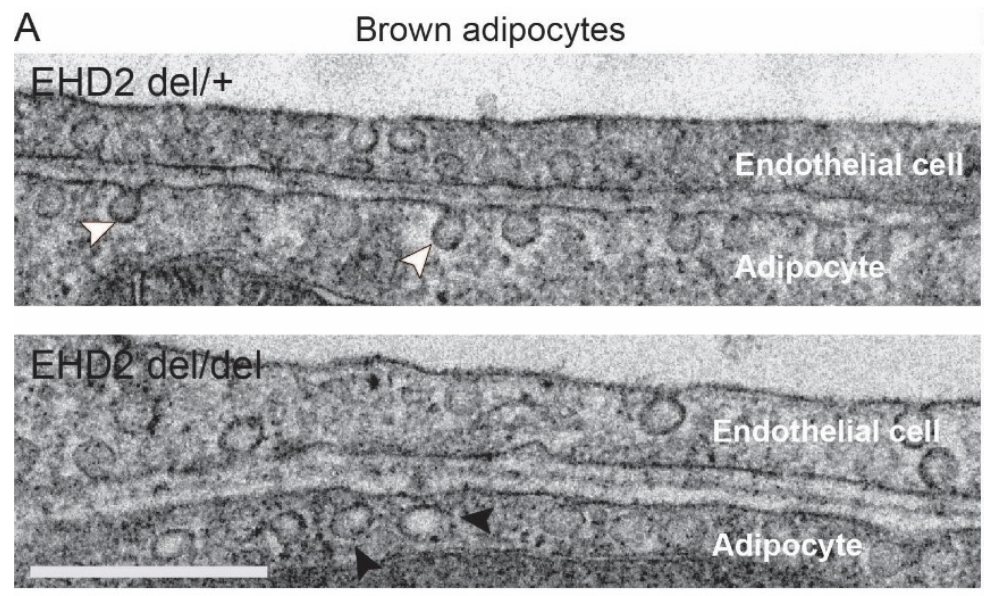

C
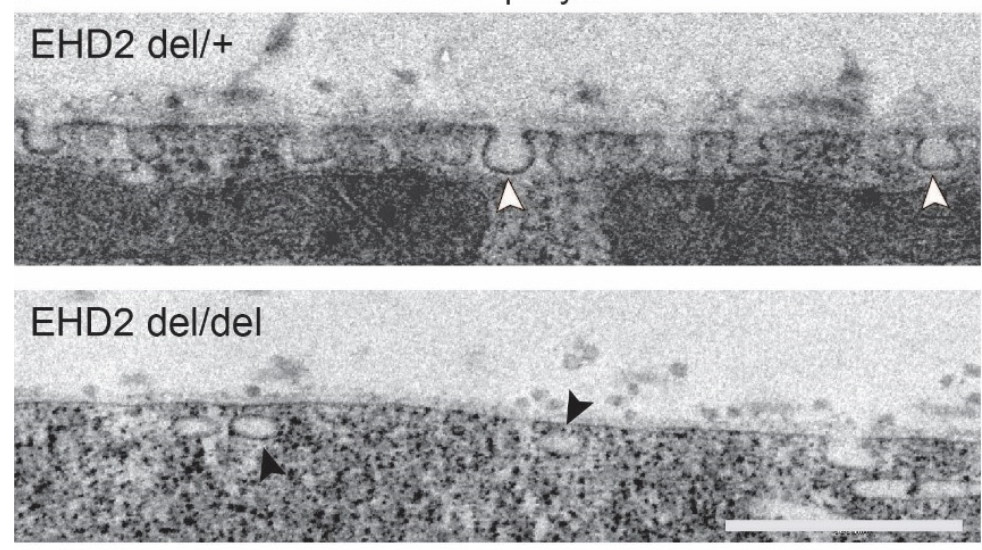

E

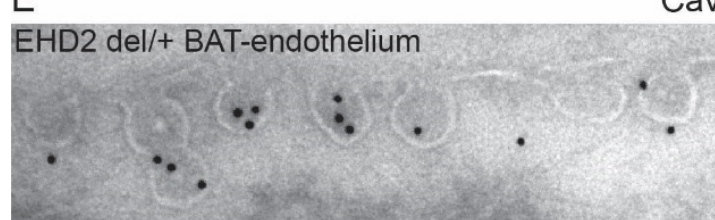

F Electron tomography

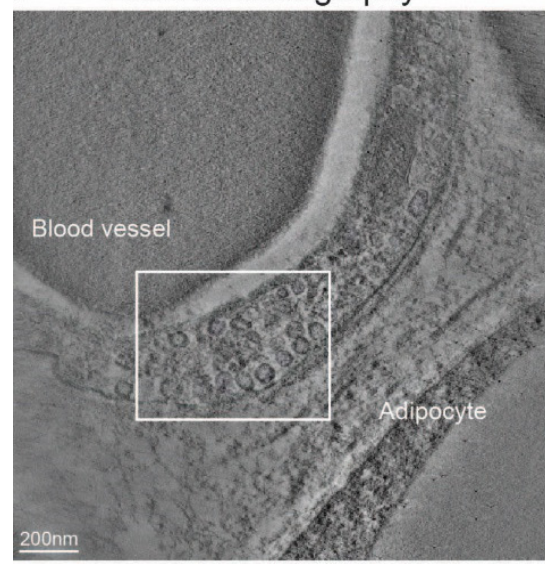

EHD2 del/del brown adipocyte

G
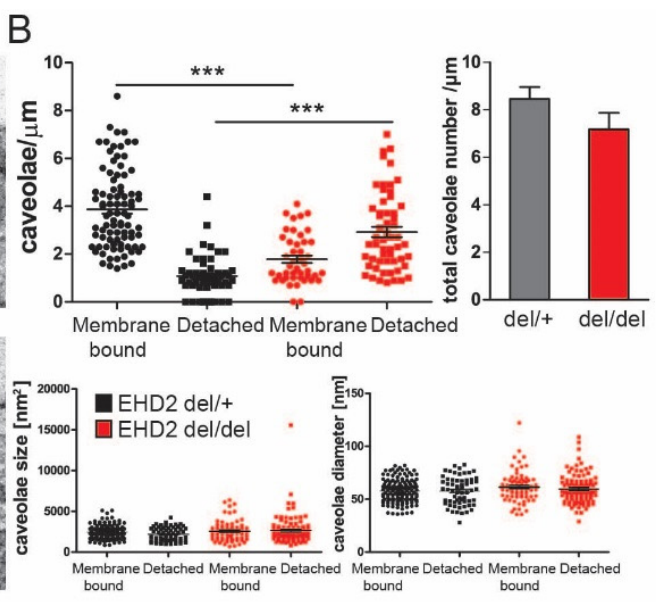

D
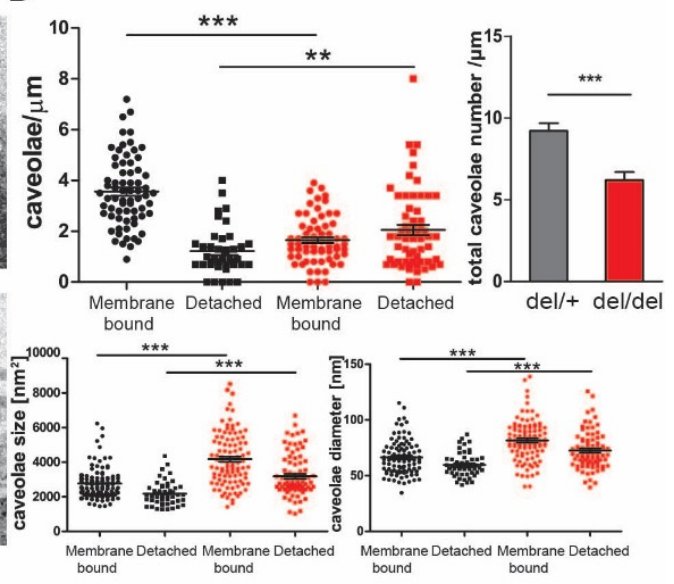

EHD2 del/del BAT-endothelium

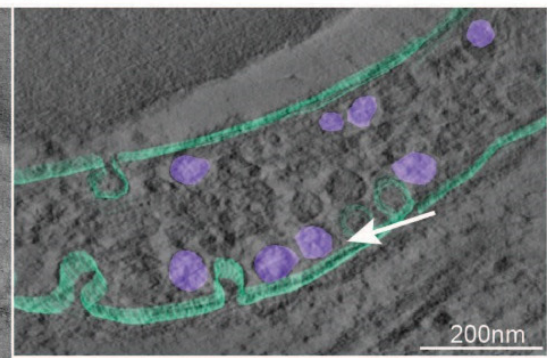

Detached caveolae

Plasma

membrane
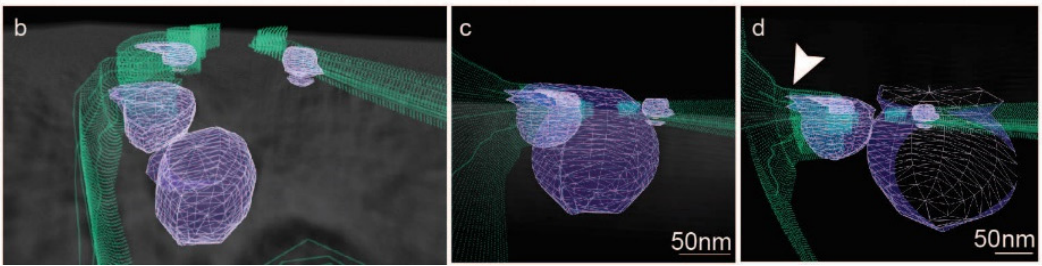

Fig. 3: Loss of EHD2 resulted in detached caveolae in vivo 
540 A-B Representative EM images of BAT from EHD2 del/+ and del/del mice and systematic analysis (caveolae 541 number: $\mathrm{n}($ del $/+)=140 / 3, \mathrm{n}($ del $/$ del $)=100 / 3$; caveolae size and diameter: $\mathrm{n}(\mathrm{del} /+)=201 / 3, \mathrm{n}(\mathrm{del} / \mathrm{del})=$ 542 171/3). Scale bar $500 \mathrm{~nm}$.

543 C-D EM images of EHD2 del/+ and del/del WAT (caveolae number: $\mathrm{n}(\mathrm{del} /+$ ) = 108/3, $\mathrm{n}(\mathrm{del} / \mathrm{del})=124 / 3$;

544 caveolae size and diameter: $\mathrm{n}(\mathrm{del} /+)=151 / 3, \mathrm{n}(\mathrm{del} / \mathrm{del})=185 / 3)$. Scale bar $500 \mathrm{~nm}$.

545 E Representative image for EM gold immunolabeling against Cav1. Control labeling did not reveal specific 546 staining. Scale bar $200 \mathrm{~nm}$.

547 F-G Electron tomogram of a $150 \mathrm{~nm}$ EHD2 del/del BAT section (F). The 3D model contains the plasma 548 membrane ( $G$, green) and the detached caveolae (violet). Detachment of caveolae was observed by 549 changing the viewing angle (white arrow indicates the direction). Closer inspection of cell membrane and 550 caveolae clearly showed displacement of caveolae from the membrane. The 3D model also revealed 551 attachment of caveolae to the membrane (arrow head).

552 Graphs illustrate each replicate with mean +/- SE, column bar graphs illustrate mean + SE t-test or Mann 553 Withney $U$ test were used to calculate significance, ${ }^{* *} \mathrm{P}<0.001 ;{ }^{* * *} \mathrm{P}<0.0001$. See also Movie $\mathrm{S} 1$ 

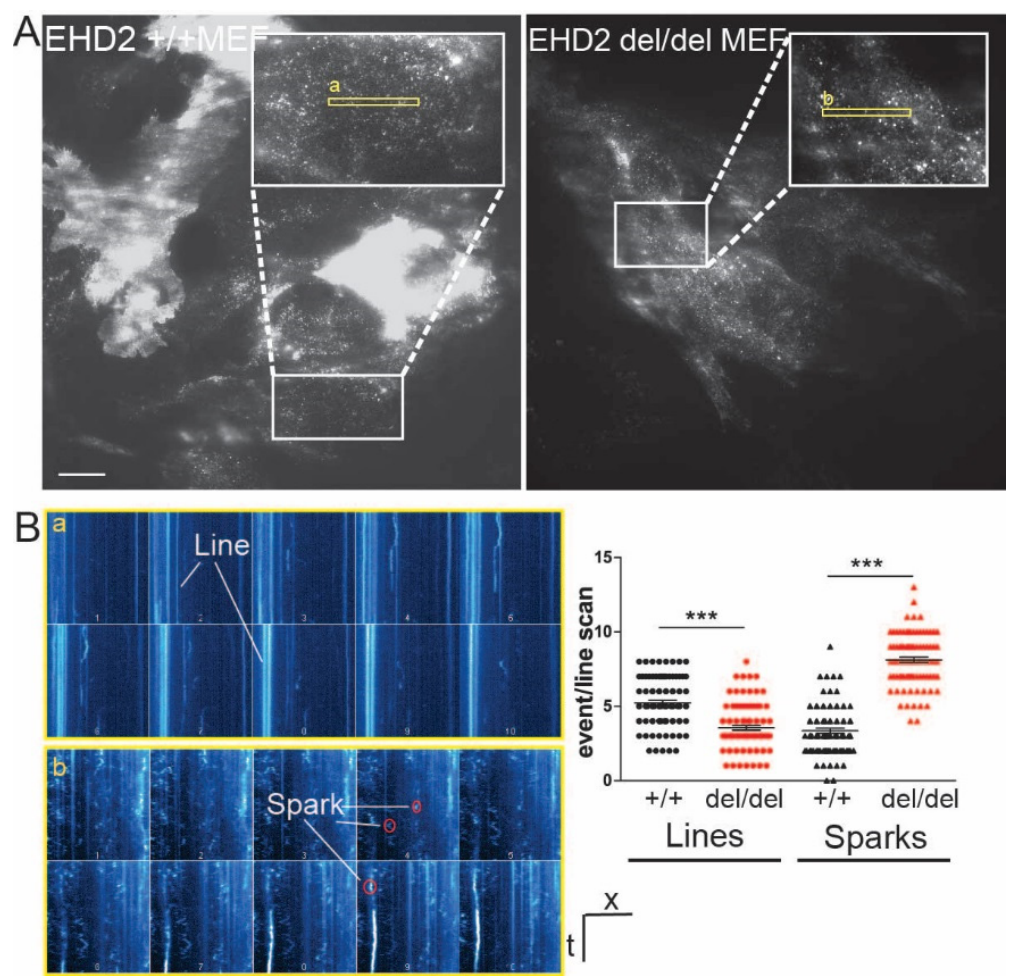

\section{Fig. 4 Enhanced caveolar mobility in cells lacking EHD2}

557 A-B TIRF live-imaging of EHD2 +/+ and del/del MEFs expressing pCav1-EGFP. Line scan analysis of the recorded Cav1 intensities revealed for fixed, non-moving caveolae lines and for fast moving caveolae

559 single sparks (as illustrated in a and b, $n(+/+)=90 / 3 ; n($ del/del) $=92 / 3$; each replicate is represented 560 with mean $+/-\mathrm{SE}$ ), ${ }^{* * *} \mathrm{P}<0.0001$. See also Fig. S6 and Movie S2-4. 

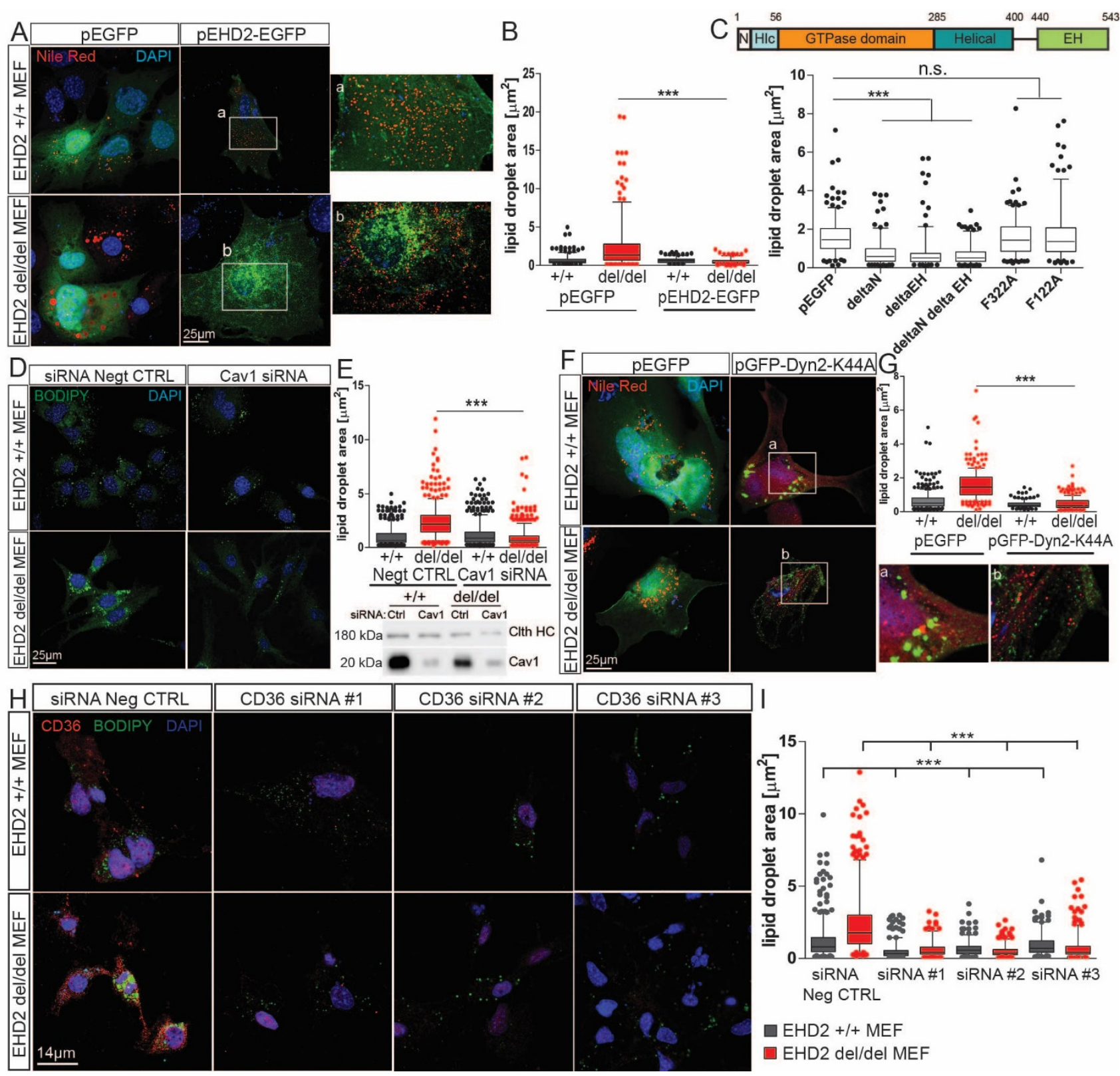

Fig. 5: EHD2-mediated fatty acid uptake depends on Cav1, Dyn2 and CD36

A-B EHD2 +/+ and del/del MEFs were transfected with either pEGFP or pEHD2-EGFP, incubated for $48 \mathrm{~h}$ and afterwards treated for $6 \mathrm{~h}$ with oleic acid and Nile Red staining was performed to determine LDs (pEGFP: $\mathrm{n}(+/+)=309 / 3, \mathrm{n}(\mathrm{del} / \mathrm{del})=310 / 3 ;$ pEHD2-EGFP: $\mathrm{n}(+/+)=218 / 4, \mathrm{n}($ del/del) = 184/4).

567 Red staining was performed to determine LDs $(n(p E G F P)=275 / 3 ; n(p E H D 2-$ deltaN-EGFP) $=193 / 3$;

$568 \mathrm{n}(\mathrm{pEHD} 2$-deltaEH-EGFP $)=197 / 3 ; \mathrm{n}(\mathrm{pEHD} 2$-deltaN-EH-EGFP $)=196 / 3 ; \mathrm{n}(\mathrm{pEHD2}-\mathrm{F322A}-\mathrm{EGFP})=204 / 3$;

$569 n(p E H D 2-F 122 A-E G F P)=212 / 3)$.

570 D-E EHD2 del/del MEFs were treated with Cav1 siRNA and lipid droplets were stained with BODIPY

571 (negative control: $\mathrm{n}(+/+)=504 / 3, \mathrm{n}(\mathrm{del} / \mathrm{del})=530 / 3$; Cav1 siRNA: $\mathrm{n}(+/+$ ) = 521/3, $\mathrm{n}(\mathrm{del} / \mathrm{del})=558 / 3$ ); Clth

572 HC - clathrin heavy chain. 
573 F-G EHD2 +/+ and del/del MEFs were transfected with either pGFP-Dyn2-K44A, incubated for $18 \mathrm{~h}$ and

574 afterwards treated for $6 \mathrm{~h}$ with oleic acid and Nile Red staining was performed to determine LDs (pEGFP:

$575 \mathrm{n}(+/+)=309 / 3, \mathrm{n}(\mathrm{del} / \mathrm{del})=233 / 4 ;$ pGFP-Dyn2-K44A: $\mathrm{n}(+/+)=136 / 3, \mathrm{n}($ del $/$ del $)=237 / 4)$.

576 H-I LD size after CD36 siRNA knockdown in EHD2 +/+ and del/del MEFs ( $D$, negative control: $\mathrm{n}(+/+)=584 / 6$,

$577 \mathrm{n}(\mathrm{del} / \mathrm{del})=475 / 6 ;$ CD36 siRNA\#1: $\mathrm{n}(+/+)=341 / 3, \mathrm{n}(\mathrm{del} / \mathrm{del})=249 / 3 ; \operatorname{CD} 36$ siRNA\#2: $\mathrm{n}(+/+)=412 / 3$,

$578 \mathrm{n}(\mathrm{del} / \mathrm{del})=468 / 3 ;$ CD36 siRNA\#3: $\mathrm{n}(+/+)=251 / 3, \mathrm{n}(\mathrm{del} / \mathrm{del})=368 / 3$; graph illustrates each replicate with

579 mean +/- SE, 2-way ANOVA test were used to calculate significance between siRNA negative CTRL and

580 siRNA, t-test was used between $+/+$ and del/del data).

581 Box plots indicate mean $+/$ - SE and single replicates of $5 \%$ of maximal and minimum values are illustrated,

582 t-test or Mann Withney $U$ test were used to calculate significance, $* * * P<0.0001$. See also Fig. $S 7$.

583

584 

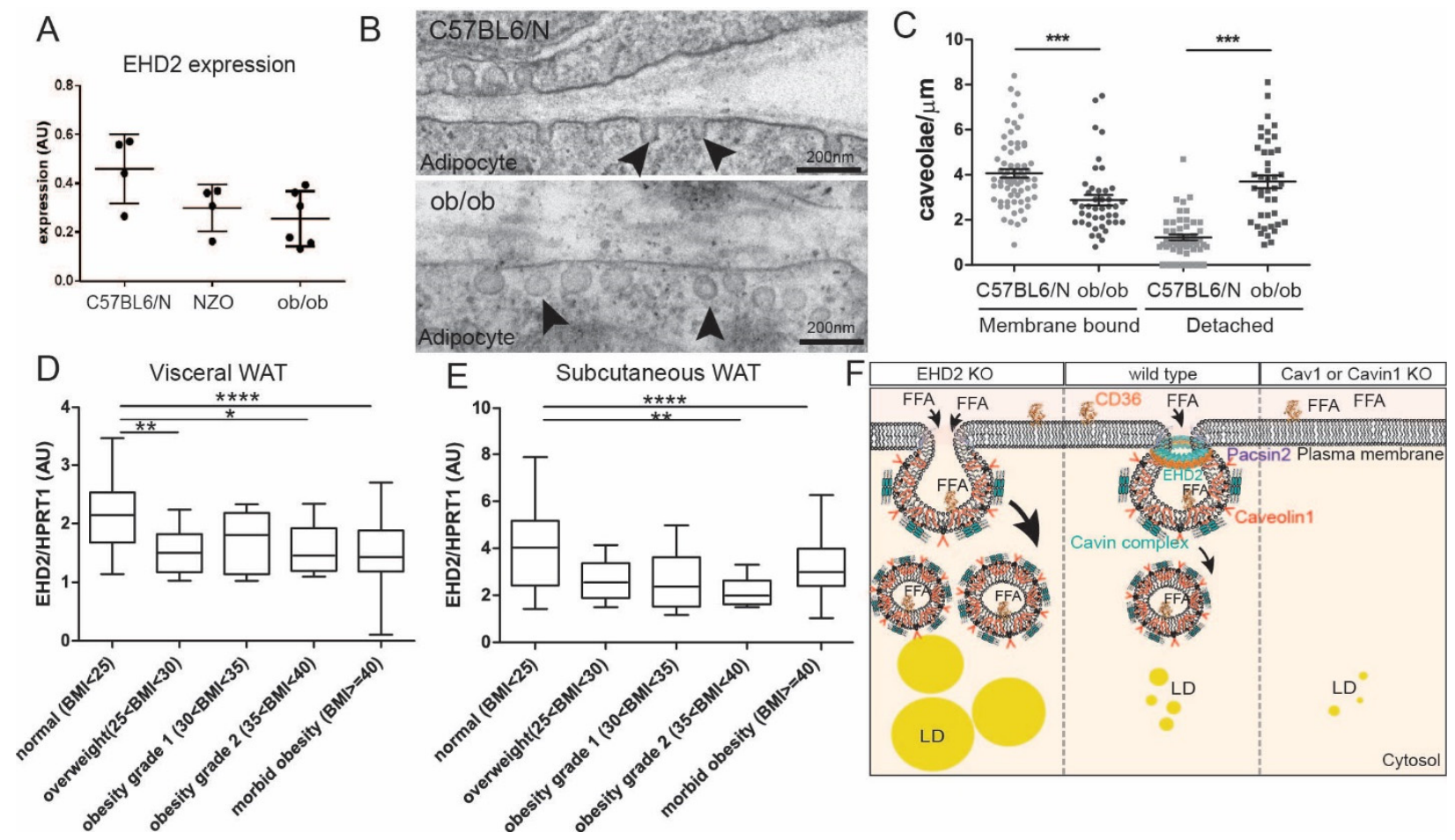

587

Fig. 6: Decreased EHD2 expression in diet induced obesity

588

A EHD2 expression level was analyzed in fat tissue of ob/ob or NZO mouse models compared to C57BL6/N

589 mice $(n=5)$.

590 B-C Investigation of caveolae by EM imaging ( $($ ob/ob mice) $=85 / 2 ; n(C 57 B L 6 / N)=117 / 2 ;$ ).

D-E EHD2 expression in visceral (E) or subcutaneous WAT (F) in obese patients (n(normal) = 31; $\mathrm{n}$ (overweight) $=23 ; \mathrm{n}$ (obesity grade 1 ) $=7 ; \mathrm{n}$ (obesity grade 2 ) $=17 ; \mathrm{n}$ (obesity grade 3 ) $=202$ )

593 F The model illustrates how fatty acid uptake is affected by caveolae function. In the absence of caveolae,

594 lipid uptake is reduced resulting in smaller lipid droplets compared to normal conditions. In the absence

595 of EHD2, lipid uptake is increased, suggesting a regulatory function of EHD2 in caveolae-dependent lipid 596 uptake. FFA - Free fatty acid, LD - lipid droplet.

597 Box plots indicate median with whiskers from maximal to minimum value, or each replicate with mean 598 +/- SE is represented, normal distributed groups were analyzed by t-test, not normal distributed values 599 with Mann Withney U test, * $\mathrm{P}<0.05, * * * \mathrm{P}<0.0001$. 


\section{Supporting Information}

602 Extended Material and Methods

603 Mouse embryonic fibroblast isolation and immortalization. All animals were handled accordingly to governmental animal welfare guidelines. MEFs were obtained from E14.5 EHD2 +/+ or del/del embryos.

605

606

607

608

609

610

611

612

613

614

615

616

617

618

619

620

621

622

623

624

625

626

627

628

629

630

Therefore female, pregnant EHD2 del/+ were sacrificed by cervical dislocation, the embryos were dissected and removed from the yolk sac in sterile, cold PBS. For genotyping, a small piece of each mouse embryo tail was harvested followed by complete dissection of the whole embryo. Afterwards, the embryo pieces were treated with $0.25 \%$ trypsin/EDTA (Sigma) over night at $4{ }^{\circ} \mathrm{C}$. After aspiration of the trypsin solution, $10 \mathrm{ml}$ culture medium (DMEM/10\%FBS/5\% penicillin/streptomycin) was added and tissue pieces were break up by pipetting. The cell suspension was transferred in $75 \mathrm{~cm}^{2}$ culture flask for cultivation at $37{ }^{\circ} \mathrm{C}$ and $5 \% \mathrm{CO}_{2}$. Immortalization of isolated primary MEFs was assured by frequently splitting. From passage 15 an increased growth rate was observed suggesting immortalized MEFs. For all experiments MEFs between passage 12 and 32 was used. For LD growth, MEFs were either treated with $10 \mu \mathrm{g} / \mathrm{ml}$ insulin, $2.5 \mathrm{mM}$ dexamethasone, $50 \mathrm{mM} \mathrm{IBMX}$ and $25 \mathrm{mM}$ rosiglitazone (all obtained from Sigma) diluted in culture medium (differentiation medium) or $0.016 \mathrm{M}$ oleic acid and $10 \mu \mathrm{g} / \mathrm{ml}$ insulin diluted in DMEM. Primary adipocyte cell culture. Male EHD2 del/+ and EHD2 del/del mice or EHD2 cKO flox/wt or flox/flox were sacrificed by cervical dislocation and gonadal WAT was removed. Adipocytes and stromal vascular fraction (SVF) were isolated after washing the tissue in sterile PBS and digestion by collagenase type II (Sigma C6885). Mature adipocytes floating in the upper phase were transferred in a new flask and diluted with culture medium (DMEM/10\%FBS/5\% penicillin/streptomycin), SVF was obtained after 5 min centrifugation at 1,000 rpm. After complete tissue break up the adipocyte cell suspension was passed through a $270 \mu \mathrm{m}$ cell strainer and the cells were plated in $75 \mathrm{~cm}^{2}$ culture flask at $37{ }^{\circ} \mathrm{C}$ and $5 \% \mathrm{CO}_{2}$ whereby pre-adipocytes adhere to the flask and mature adipocytes float in the medium. SVF suspension was cleaned by passing through $70 \mu \mathrm{m}$ cell strainer. The following day the culture medium was exchanged to remove dead or non-adherent cells. After 5 days, both pre-adipocytes and SVF were split by $0.25 \%$ trypsin/EDTA solution and merged for further cultivation. Differentiation to mature adipocytes was induced by $10 \mu \mathrm{g} / \mathrm{ml}$ insulin, $2.5 \mathrm{mM}$ dexamethasone, $50 \mathrm{mM} \mathrm{IBMX}$ and $25 \mathrm{mM}$ rosiglitazone diluted in culture medium. If not otherwise mentioned the primary pre-adipocytes were incubated for 5 days with differentiation medium and medium was changed after 2 days. Delipidation of FBS was carried out as described by (1). EHD2 cKO adipocytes were transfected with Cre recombinase-EGFP by using adeno- 
631

632

633

634

635

636

637

638

639

640

641

642

643

644

645

646

647

648

649

650

651

652

653

654

655

656

657

658

659

660

661

662

associated virus particles 8 (AAV8) produced from pAAV.CMV.HI.eGFP-Cre.WPRE.SV40 (addgene, \#105545). The adipocyte cell culture was transfected and differentiated for 5 days.

Oil Red $\mathbf{O}$ staining. LDs in tissue sections or cultivated adipocytes and MEFs were stained with Oil Red $\mathrm{O}$ (Sigma \#00625) as published by (2). Briefly, freshly dissected liver of muscle pieces were frozen in liquid nitrogen, embedded in TissueTek and $10 \mu \mathrm{m}$ cryostat sections (Leica) were prepared. After fixation with 4\% para-formaldehyde (PFA, Merck) freshly prepared Oil Red O staining solution was applied for $10 \mathrm{~min}$. The sections were washed with PBS and embedded in ImmoMount (Invitrogen). Cultivated cells were fixed, treated with 60\% isopropanol (Merck) for $2 \mathrm{~min}$ and then incubated with Oil Red O staining solution for $5 \mathrm{~min}$. After washing with water until complete removal of Oil Red $\mathrm{O}$ the stained cells or sections were analyzed by Zeiss Axiovert microscope (20x Zeiss objective). Staining intensity was measured with ImageJ. Histology. EHD2 del/+ and EHD2 del/del mice were anesthetized with 2\% ketamine/10\%rompun, perfused first by $30 \mathrm{ml}$ PBS and next by $50 \mathrm{ml}$ 4\% PFA and tissues were dissected. After $24 \mathrm{~h}$ of fixation in $4 \%$ PFA, tissues were dehydrated in 3 steps (each $24 \mathrm{~h}$ ) from $70-100 \%$ EtOH and afterwards incubated in xylol (Merck) for $48 \mathrm{~h}$. Next, the tissues were embedding in liquid paraffin at ca. $65^{\circ} \mathrm{C}$ and cooled down on ice. $4 \mu \mathrm{m}$ paraffin sections were obtained, de-paraffinized and hydrated and Masson Trichrome staining (Kit, Sigma) was applied. Briefly, sections were stained with Bouin solution for $15 \mathrm{~min}$ at $60^{\circ} \mathrm{C}$, followed by Haematoxylin Gill No. 2 staining for 5 min and incubation in Biebrich-Scarlet-Acid Fuchsin for 5 min. Next, the tissue sections were treated with Phosphotungstic/Phosphomolybdic Acid Solution and Aniline Blue solution both for $5 \mathrm{~min}$, and acetic acid treatment (1\%) for $2 \mathrm{~min}$. After extensive washing the sections were dehydrated, incubated in xylol and embedded with Roti Histo Kit (Carl Roth). Images were obtained at Zeiss Axiovert100 microscope.

Immunohistostaining of cryostat sections. Perfused and fixated EHD2 del/+ and EHD2 del/del mice (as described before) were dissected and the investigated tissue pieces were further fixed for 1-4 $\mathrm{h}$ in $4 \%$ PFA, transferred to $15 \%$ sucrose (in PBS, Merck) for $4 \mathrm{~h}$ and finally incubated overnight in $30 \%$ sucrose. After embedding in TissueTek, the tissue is frozen at $-80^{\circ} \mathrm{C}$. 5-15 $\mu \mathrm{m}$ sections were obtained in a cryostat at $20--30^{\circ} \mathrm{C}$ and stored at $-20^{\circ} \mathrm{C}$. For immunostainings, the cryostat sections were incubated with blocking buffer ( $1 \%$ donkey serum/1\% TritonX100/PBS) for $1 \mathrm{~h}$ at room temperature, and treated overnight at $4^{\circ} \mathrm{C}$ with the first antibody diluted in blocking buffer. After washing with PBS/1\%Tween, the secondary antibody was applied for $2 \mathrm{~h}$ at room temperature. After completion of the staining, the sections were washed carefully and embedded in ImmoMount. The stained sections were analyzed with Zeiss LSM700 microscope provided with Zeiss objectives 5, 10, 20, 40 and 63x. The obtained images were further investigated by ZEN software and ImageJ/Fij. 
663

664

665

666

667

668

669

670

671

672

673

674

675

676

677

678

679

680

681

682

683

684

685

686

687

688

689

690

691

692

693

694

Transfection and siRNA knockdown. Cultivated MEFs were transfected with the following plasmids pEHD2-EGFP, pEHD2-deltaN-EGFP, pEHD2-deltaEH-EGFP, pEHD2-deltaN-EH-EGFP, pEHD2-F322A-EGFP, pEHD2-F122A-EGFP, pCav1-EGFP (provided from R.L.), pGFP-Dyn2-K44A (provided from M.L., addgene \#22301) or pEGFP by lipofectamine 3000 (Invitrogen) accordingly to the manufacture's protocol. Transfected cells were incubated for $48 \mathrm{~h}$ and afterwards the treated cells were analyzed by confocal microscopy or TIRF. siRNA knockdown of CD36 or Cav1 was performed in freshly split MEFs by electroporation with the GenePulser XCell (Biorad). Briefly, MEFs were split as described before and the obtained cell pellet was resuspended in OptiMEM (Gibco). After cell counting, the MEF cell suspension was diluted to $1.5 \times 10^{6}$ cells $/ \mathrm{ml}$ and $300 \mu$ l were transferred into electroporation cuvettes $(2 \mathrm{~mm}$, Biorad). CD36 or Cav1 stealth siRNA and siRNA negative control (medium GC content, CD36 siRNA\#1 GGAAUUUGUCCUAUUGGCCAAGCUA, CD36 siRNA\#2 CCAAGUCUUCUAUGUUCCAAACAAG; CD36 siRNA\#3 CCAAUAACUGUACAUCUUAUGGUGC) was added to a final concentration of $200 \mathrm{nM}$. After carful mixing, the cuvettes were placed into the electroporation device and the pulse ( $160 \mu \mathrm{OHM}, 500 \mu \mathrm{F}, \infty$ resistance) was applied. The electroporated cells were cultivated in DMEM/10\%FBS for $48 \mathrm{~h}$ before the experiments were started. Successful siRNA knockdown was monitored by CD36 antibody staining.

TIRF live imaging of caveolae movement. MEFs transfected with pCav1-EGFP were incubated for $48 \mathrm{~h}$ on fibronectin coated cover slips (25 mm diameter). Samples were mounted in Attofluor Cell Chamber (Thermo) in a physiological buffer ( $130 \mathrm{mM} \mathrm{NaCl}, 4 \mathrm{mM} \mathrm{KCl}, 1.25 \mathrm{mM} \mathrm{NaH}_{2} \mathrm{PO}_{4}-\mathrm{H}_{2} \mathrm{O}, 25 \mathrm{mM} \mathrm{NaHCO}, 10$ $\mathrm{mM}$ glucose, 2 mM CaCl $2,1 \mathrm{mM} \mathrm{MgCl}$, pH 7.3, 305-315 mOsm/kg).

TIRF imaging was performed on an inverted Microscope (Nikon Eclipse Ti) equipped with a 488 laser (Toptica), an dicroic mirror (AHF, zt405/488/561/640 rpc), a 60x TIRF objective (Nikon, Apo TIRF NA 1.49), an appropriate emission filter (AHF, 400-410/488/561/631-640) and a sCMOS camera (mNeo, Andor). All components were operated by open-source ImageJ-based micromanager software. All experiments were performed at $37^{\circ} \mathrm{C}$. To investigate the movement of single caveolae transfected cells were selected in which regions of individual Cav1 spots were observed (ROls illustrated in Fig. 4A, enhanced images). Recordings were obtained with the following imaging settings: image size 1776x1760 pixel, 1x1 binning, 500 frames, 200 ms exposure time/frame. For data analysis only the first 150 frames were investigated. After cropping to the specific ROI, kymograph analysis of several positions within the ROls were carried out using the Reslice function of ImageJ/Fij. Carefully investigation of the kymographs revealed a single, straight line for fixed, not moving caveolae and sparks or short lines for fast moving caveolae.

Transmission Electron microscopy (TEM). Mice were fixed by perfusion with $4 \%(w / v)$ formaldehyde in $0.1 \mathrm{M}$ phosphate buffer and tissues were dissected to $1-2 \mathrm{~mm}^{3}$ cubes. For morphological analysis, tissue 
blocs were postfixed in phosphate buffered $2.5 \%(\mathrm{v} / \mathrm{v})$ glutaraldehyde. Samples were treated with $1 \%$ $(\mathrm{v} / \mathrm{v})$ osmium tetroxide, dehydrated in a graded series of ethanol and embedded in the PolyBed ${ }^{\circledR} 812$ resin (Polysciences Europe $\mathrm{GmbH})$. Ultrathin sections $(60-80 \mathrm{~nm}$ ) were cut (Leica microsystems) and stained with uranyl acetate and lead citrate before image acquisition. For immuno-labeling, samples were fixed by perfusion as described above, but postfixed in phosphate buffered $4 \%(w / v)$ formaldehyde with $0.5 \%$ (v/v) glutaraldehyde for 1 hour. Samples were further processed as described in Slot and Geuze (Nature protocols, 2007). Briefly, samples were infiltrated with $2.3 \mathrm{M}$ sucrose, frozen in liquid nitrogen and sectioned at cryo temperatures. Sections were blocked and washed in PBS supplemented with 1\% BSA and 0.1\% glycine. Labeling was performed with an anti-caveolin-1 antibody 1:500 (abcam \#2910) and 12 $\mathrm{nm}$ colloidal gold (Dianova). Sections were contrasted with $3 \%$ tungstosilicic acid hydrate $(\mathrm{w} / \mathrm{v})$ in $2.8 \%$ polyvinyl alcohol (w/v) (3). Samples were examined at $80 \mathrm{kV}$ with a Zeiss EM 910 electron microscope (Zeiss). Acquisition was done with a Quemesa CDD camera and the iTEM software (Emsis GmbH).

In situ hybridization. Digoxigenin-labeled riboprobes were generated using a DIG-RNA labeling kit (Roche). In situ hybridizations were performed on $14 \mu \mathrm{m}$ cryosections prepared from E18.5 wt embryos as previously described (4). To generate an Ehd2 specific in situ probe, a $400 \mathrm{bp}$ fragment was amplified from wildtype cDNA using PCR and primer listed below. The PCR product was cloned into pGEM-Teasy (Promega). T7 and sp6 polymerases were used to generate Ehd2-sense and antisense probes, respectively. EDH2_ISH_FWD: 5'-CAGGTCCTGGAGAGCATCAGC-3'; EDH2_ISH_REV: 5'GAGGTCCTGTTCCTCCAGCTCG-3'

Western Blot. EHD2 protein level in different tissues was examined by Western Blot. Therefore EHD2 +/+, EHD2 del/+ and EHD2 del/del mice were sacrificed by cervical dislocation and organs were dissected and snap frozen in liquid nitrogen. After homogenization of the tissue in 1x RIPA buffer (Abcam) with a glass homogenizer, the tissue lysate was incubated for $1 \mathrm{~h}$ on ice followed by $15 \mathrm{~min}$ centrifugation at 15,000 rpm. Supernatant was transferred in a fresh tube and protein concentration was measured by NanoDrop. At least $10 \mu \mathrm{g}$ protein/lane was applied to 4-12\% SDS-PAGE NuPage (Invitrogen) and SDS-PAGE was performed accordingly to the manufacture's protocol. Afterwards, proteins were blotted on nitrocellulose membrane (Amersham) at $80 \mathrm{~V}$ for $1 \mathrm{~h}$, followed by blocking of the membranes with $5 \%$ milk powder (in TBST, $150 \mathrm{mM} \mathrm{NaCl}, 20 \mathrm{mM}$ Tris- $\mathrm{HCl}, \mathrm{pH}$ 7.5, 0.1\% Tween20) for $2 \mathrm{~h}$ at room temperature. To detect EHD2 protein level rabbit-anti-EHD2 $(1: 2,000)$ was applied over night at $4{ }^{\circ} \mathrm{C}$. After washing with TBST the secondary antibody goat-anti-rabbit-HRP was added to the membrane for $2 \mathrm{~h}$ at room temperature. Detection of EHD2 bands results from ECL detection solution and intensities were obtained by ChemiDoc XRS (Biorad). 
Antibodies. Anti-EHD2-Rb (self-made), anti-Cav1-Mouse (BD Biosciences \#610407), anti-Rabbit IgG HRP (dianova), anti-Mouse IgG HRP (dianova), anti-Perilipin1-Rb (Cell signaling \#9349), anti-CD36-Rb (Novus Bio \#NB400-144 and abcam \#ab133625), anti-Cavin1-Rb (Abcam, \#ab48824), anti-Rb-Cy3 (dianova), antimouse IgG-Alexa488 (ThermoScientific \#R37114), DAPI (Sigma D9542)

Blood plasma analysis. To measure distinct blood plasma parameter related to metabolic changes like adiponectin, insulin or free fatty acids blood was taken from EHD2 del/+ and EHD2 del/del mice immediately after cervical dislocation. All blood samples were taken at $10.00 \mathrm{am}$. Briefly, mice were opened and the thorax was partly removed to get access to the left heart ventricle, a cannula was inserted and blood samples were taken. After short centrifugation at high speed, the plasma fraction was transferred to a fresh tube and snap frozen in liquid nitrogen. The following assays were used to measure the described blood plasma markers: Plasma insulin levels were measured by Mouse Ultrasensitive Insulin ELISA (80-INSMSU-E10, Alpco). Plasma adiponectin and leptin levels were measured by Mouse Adiponectin/Acrp30 (DY1119) and Mouse/Rat Leptin (MOB00) ELISA kits (R\&D Systems). Plasma lipids were quantified with commercially available kits: cholesterol (Cholesterol liquicolour colorimetric assay, Human, Wiesbaden, Germany), triglycerides/glycerol (Triglyceride/Glycerol Calorimetric Assay, Sigma) and non-esterified fatty acids (Wako Chemicals). All measurements were done according to manufacturers' recommendations.

Fatty acid uptake assay. EHD2 del/+ and EHD2 del/del pre-adipocytes were seeded in 6-well plates (100.000 cells/well) and differentiated in mature adipocytes as described above. The fatty acid uptake assay was performed as described elsewhere (5). Briefly, after 5 days of differentiation, adipocytes were starved for $1 \mathrm{~h}$ with serum-free DMEM. Next, $2 \mu \mathrm{M}$ dodecanoic acid (FA12) labelled with BODIPY (Molecular probes \#D3822) diluted in serum-free DMEM + $10 \mu \mathrm{g} / \mathrm{ml}$ insulin was added to the adipocytes and incubated for 5, 10, 20, 30 and $60 \mathrm{~min}$ at $37^{\circ} \mathrm{C}$. After washing twice with ice-cold PBS, $150 \mu \mathrm{l} 0.25 \%$ trypsin/EDTA/PBS was applied to detach the cells. The adipocytes were treated with $500 \mu$ l ice-cold FACS buffer (HBSS/10\%FBS/10 mM EDTA) and the cell solution was transferred to FACS tubes. Shortly before measurement, $1 \mu \mathrm{l} / \mathrm{ml}$ propidium iodide was added. FACS experiments were performed at LSR Fortessa 5Laser with the following parameters: FSH: A, H, W, Voltage 255; SSC: A, H, W, Voltage 203; A488: A, 754 Voltage 198; PE: A, Voltage 341. For each FACS sample 30.000 cells were investigated. As negative control unstained EHD2 del/+ and EHD2 del/del adipocytes were examined at first and the obtained BODIPY intensity values were used as a reference for unstained cells. To exclude adipocytes which did not show 757 any positive fatty acid uptake, all unstained cells were removed resulting in an only positive stained 758 population (R1, illustrated in red in Fig. 2F). Within this R1 population adipocytes with strongly increased 

BODIPY intensity values were gated to population R2 (blue, Fig. 2F). Detailed analysis/gating and statistics was done by using FlyingSoftware2.5.1 (Perttu Terho, Cell Imaging Core, Turku Center for Biotechnology). For each experiment 15.000 cells were analyzed and gated to the unstained, R1 or R2 population. Next, the percentage of the cells gated to the populations were calculated for every time point and illustrated in the bar graph (Fig. S4C). R2 population was investigated in more detail by normalization to the R2 cell number of EHD2 del/+ adipocytes (Fig. 2H).

Glucose uptake assay. Glucose uptake of EHD2 del/+ and EHD2 del/del adipocytes was measured as described by BioVison (2-NBDG Glucose uptake assay). Briefly, adipocytes were treated as described for fatty acid uptake assay. However, after starvation $200 \mu \mathrm{M}$ 2-NBDG (2-deoxy-2-[(7-nitro-2,1,3benzoxadiazol-4-yl) amino]-D-glucose, molecular probes \#N13195) diluted in serum-free DMEM + 10 $\mu \mathrm{g} / \mathrm{ml}$ insulin was applied to the cells followed by incubation times from 5-60 min. Staining analysis was done as mentioned for fatty acid uptake with the same FACS parameters and gating procedure whereby only one positive stained cell population was examined (R1, illustrated in Fig. S4E).

Gene expression analysis. EHD2 del/+ and EHD2 del/del adipocytes were differentiated for 5 days, washed twice with ice-cold PBS and RNA was isolated accordingly to the Qiagen protocol (RNeasy Mini Kit, Qiagen). SuperscriptIII First Strand Synthesis Kit (Invitrogen \#18080051) was used to obtain corresponding cDNA, which then was used for real-time PCR. Gene expression levels were analyzed by GoTaq q-PCR (Promega, \#A6001) Master Mix in Fast real time PCR cycler (Applied Biosystems) accordingly to instructor's protocol. To measure the relative fold change of genes in EHD2 del/del adipocytes compared to EHD2 del/+, the comparative real-time PCR method was applied whereby actin was used as reference gene.

Total mRNA from murine gonadal adipose tissue (gWAT) was extracted with RNeasy Mini Kit (QIAGEN $\mathrm{GmbH}$, Hilden) according to manufacturer's instructions. RNA was transcribed using the Moloney Murine Leukemia Virus Reverse Transcriptase (M-MLV RT, Promega) according to manufacturer's recommendations. Expression of mRNA was determined by quantitative real-time PCR on LightCycler 480 II/384 (Roche, Rotkreuz, Switzerland) using GoTaq Probe qPCR Master Mix (Promega, Madison, USA) applying TaqMan Gene Expression Assays. Target gene expression of was normalized to the mean expression of Eef2, Ppia and Actb in murine samples.

\begin{tabular}{|l|l|l|}
\hline Gene & Description & TaqMan Assay \\
\hline EHD2 & EH-domain containing 2 & Hs.PT.58.4969281 \\
\hline Ehd2 & EH-domain containing 2 & Self-designed \\
\hline Actb & Actin, beta & Self-designed \\
\hline
\end{tabular}




\begin{tabular}{|l|l|l|}
\hline Eef2 & Eukaryotic translation elongation factor 2 & Self-designed \\
\hline Ppia & Peptidylprolyl isomerase A & Mm.PT.39a.2.gs \\
\hline
\end{tabular}

Actb Left primer: TACGACCAGAGGCATACAG, Right primer: GCCAACCGTGAAAAGATGAC, Probe:

788 TTGAGACCTTCAACACCCCAGCCA, Eef2 (Integrated DNA Technologies) Left primer:

789 CACAATCAAATCCACCGCCA, Right primer: TGAGGTTGATGAGGAAGCCC, Probe:

790 TAAGCAGAGCAAGGATGGCT, Ehd2 (UPL, Roche) Left primer: CAGCTGGAGCACCACATCT, Right primer:

791 TCATGTGCCATCAACAGCTC, UPL probe: \#80

792 Lipid composition. The measurement of lipid amount and its composition in tissue samples or cells were 793 performed by Lipotype GmbH (Dresden, Germany). For this, tissue samples were homogenized 794 accordingly to the supplied Lipotype protocol and diluted samples $(1 \mathrm{mg} / \mathrm{ml})$ were frozen and analyzed by 795 Lipotype. MEF were split and the cell pellet was diluted in cold PBS to a final cell number of 30,000 cells $/ \mathrm{ml}$.

796 Statistical analysis. At first, a normality distribution test (Kolmogorov-Smirnov test) was carried out for all 797 experimental values. If the data was normally distributed, Student t-Test (two-tailed P-value) was applied, 798 otherwise Mann-Withney-Rank-Sum (two-tailed P-value) test was used to calculate the significant 799 difference between two groups. Two-way-Anova tests were used to investigate LD size after CD36 siRNA 800 knockdown, whereby for EHD2 wt and KO MEFs each CD36 siRNA\#1-3 treated cells were compared to 801 nonsense siRNA (negative control, Fig. 5I). Box plots, if not otherwise indicated in the figure legends, 802 always represents median with whiskers from minimum to maximum, column bar graphs and line graphs 803 represent mean with mean standard error of the mean (SE). Statistical calculations were carried out by 804 using Prism (GraphPad software). Distribution of LD sizes represented in histograms were also obtained 805 by using Prism. For all experiments including the examination of mice or mouse tissue or human samples, $806 \mathrm{n}$ represents the number of mice/patients which were used (Fig. 1, 3, 6, S1-5) and all analyzed 807 cryo/paraffin sections or caveolae are also indicated (e.g.: $\mathrm{n}=80$ caveolae/3 mice). In cell culture 808 experiments (Fig. 2, 4, 5, S4-7), $\mathrm{n}$ represents the number of investigated events (e.g.: lipid droplet area) 809 and the number of independently performed experiments (e.g.: $n=80$ lipid droplets $/ 3$ independent 810 experiments). The following P-values were used to indicate significant difference between two groups: * $811 \mathrm{P}<0.05 ; * * \mathrm{P}<0.001 ; * * * \mathrm{P}<0.0001$. 


\section{Supplemental Material}

\section{Movie S1: Electron tomography of EHD2 del/del BAT (related to Fig. 3)}

815 Caveolae in a $150 \mathrm{~nm}$ EHD2 del/del BAT section were investigated by electron tomography. The movie 816 includes all images obtained during the tilting from $-60^{\circ}$ to $60^{\circ}$ (image acquisition every $2^{\circ}$ ). Both, detached 817 and plasma membrane bound caveolae can be observed. For better handling during the segmentation 818 and reconstruction in IMOD, the tomogram was reflected horizontal.

819 Movie S2-3: TIRF live imaging of caveolae in EHD2 +/+ and del/del MEFs (related to Fig. 4)

820 EHD2 +/+ and del/del MEFs were transfected with pCav1-EGFP to detect single caveolae and afterwards

821 TIRF live imaging was performed. Movie S2 illustrates an example EHD2 +/+ MEF, and Movie S3 shows an 822 EHD2 del/del MEF. Notably, Cav1 spots in MEFs lacking EHD2 showed increased caveolar dynamics.

823 Movie S4: TIRF live imaging of caveolae in EHD2 del/del MEFs transfected with pEHD2-EGFP (related to $824 \quad$ Fig. 4)

825 EHD2 del/del MEFs were co-transfected with pCav1-EGFP and pEHD2-EGFP and caveolae movement was 826 observed by TIRF live imaging. Re-expression of EHD2 in EHD2 del/del MEFs strongly reduced the 827 dynamics of caveolae. 

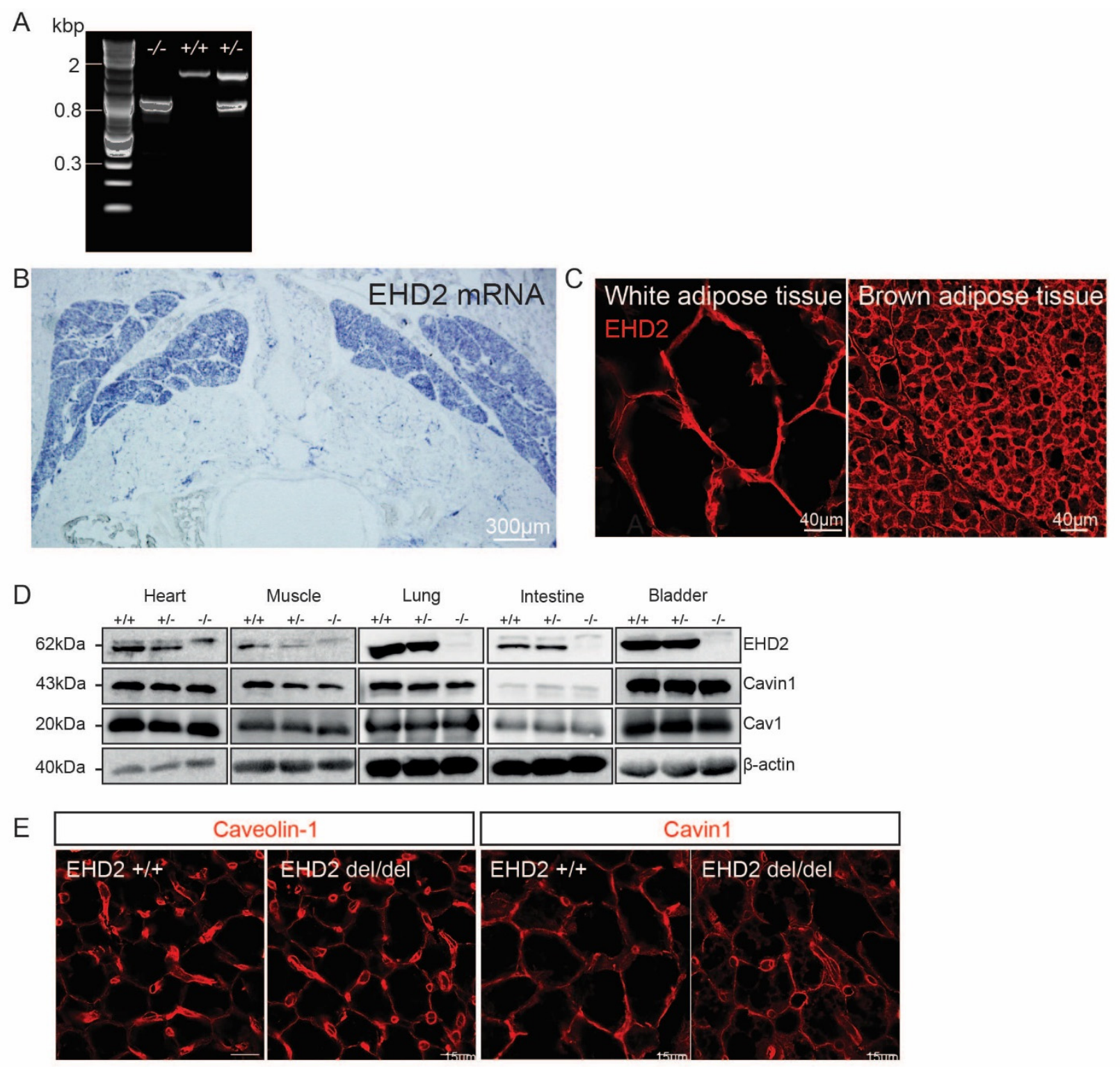

Fig. S1: EHD2, Cav1 and Cavin1 expression in BAT (related to Fig. 1)

831 A Genotyping of EHD2 delta E3 offspring (wildtype - band size 1,700 bp; EHD2 KO - band size 830 bp).

832 B In situ hybridization against EHD2 mRNA of BAT in an E18 C57BL6/N embryo.

833 C Cryostat section of adult C57BL6/N white adipose or brown adipose tissue stained against EHD2.

834 D Western Blot analysis of different tissues from EHD2+/+, +/- and -/- mice showing EHD2, Cav1 and 835 Cavin1 protein level.

836 E Cavin1 and Cav1 protein level in BAT cryostat sections from EHD2 wt and del/del mice. 

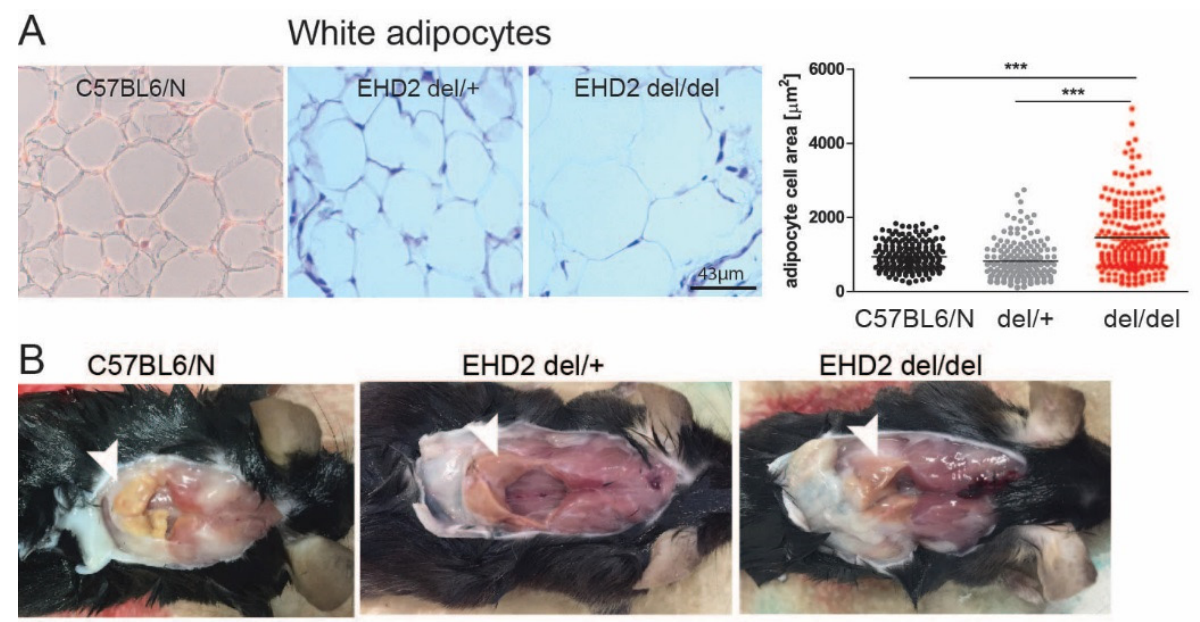

$\mathrm{EHD} 2 \mathrm{del} / \mathrm{del}$
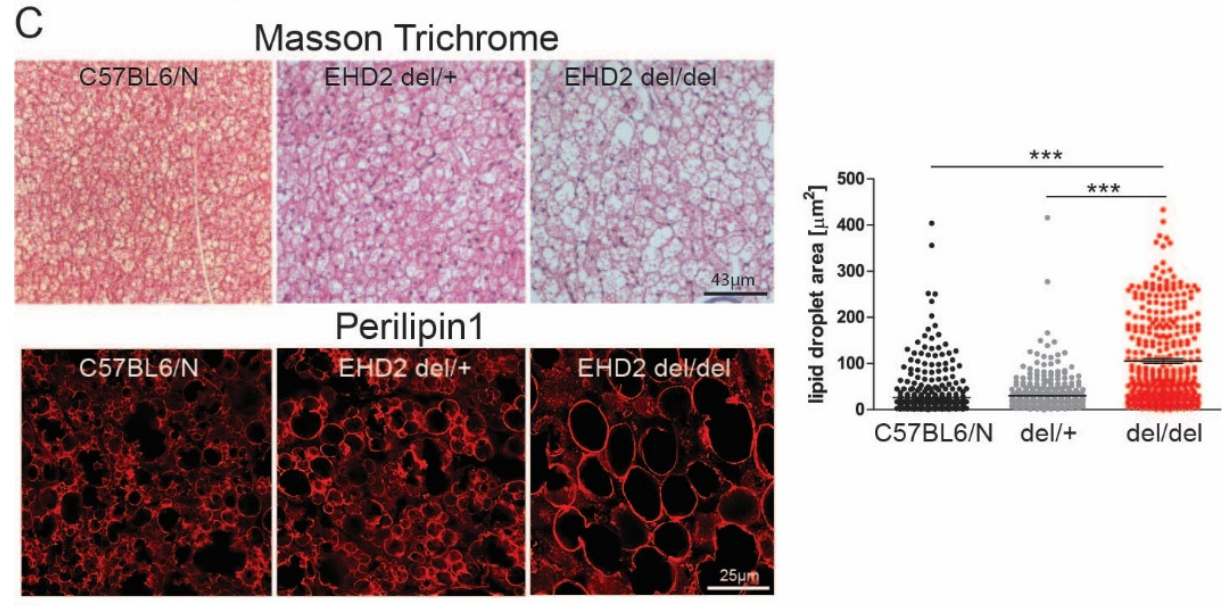

D Adipogenic gene expression

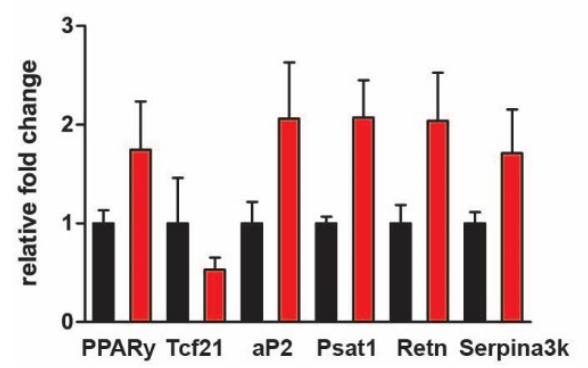

Fig. S2: C57BL6/N and EHD2 del/+ mice did not reveal any differences in lipid accumulation (related to

A WAT paraffin sections from C57BL6/N mice stained with Masson Trichrome and analyzed by adipocyte cell size $(n(C 57 B L 6 / N)=186 / 3 ; n(E H D 2 \mathrm{del} /+=172 / 3 ; n(E H D 2 \mathrm{del} / \mathrm{del})=199 / 3)$.

843 B BAT examples of C57BL6/N, EHD2 del/+ and del/del mice.

844 C BAT paraffin and cryostat sections stained against Perilipin1 obtained from C57BL6/N mice (lipid droplet size was measured by Perilipin1 staining, $n($ C57BL6/N) = 461/3; $n($ EHD2 del/+ = 398/3; $n($ EHD2 del/del $)=$ 846 352/3).

847 D Adipogenic gene expression analysis of WAT from EHD2 del/+ and EHD2 del/del mice $(n=8)$. Graphs illustrate each replicate with mean +/- SE, column bar graphs show mean $+\mathrm{SE}$, normal distributed groups were analyzed by $\mathrm{t}$-test, not normally distributed values with Mann Withney $\mathrm{U}$ test, ${ }^{*} \mathrm{P}<0.05, * * *$ 

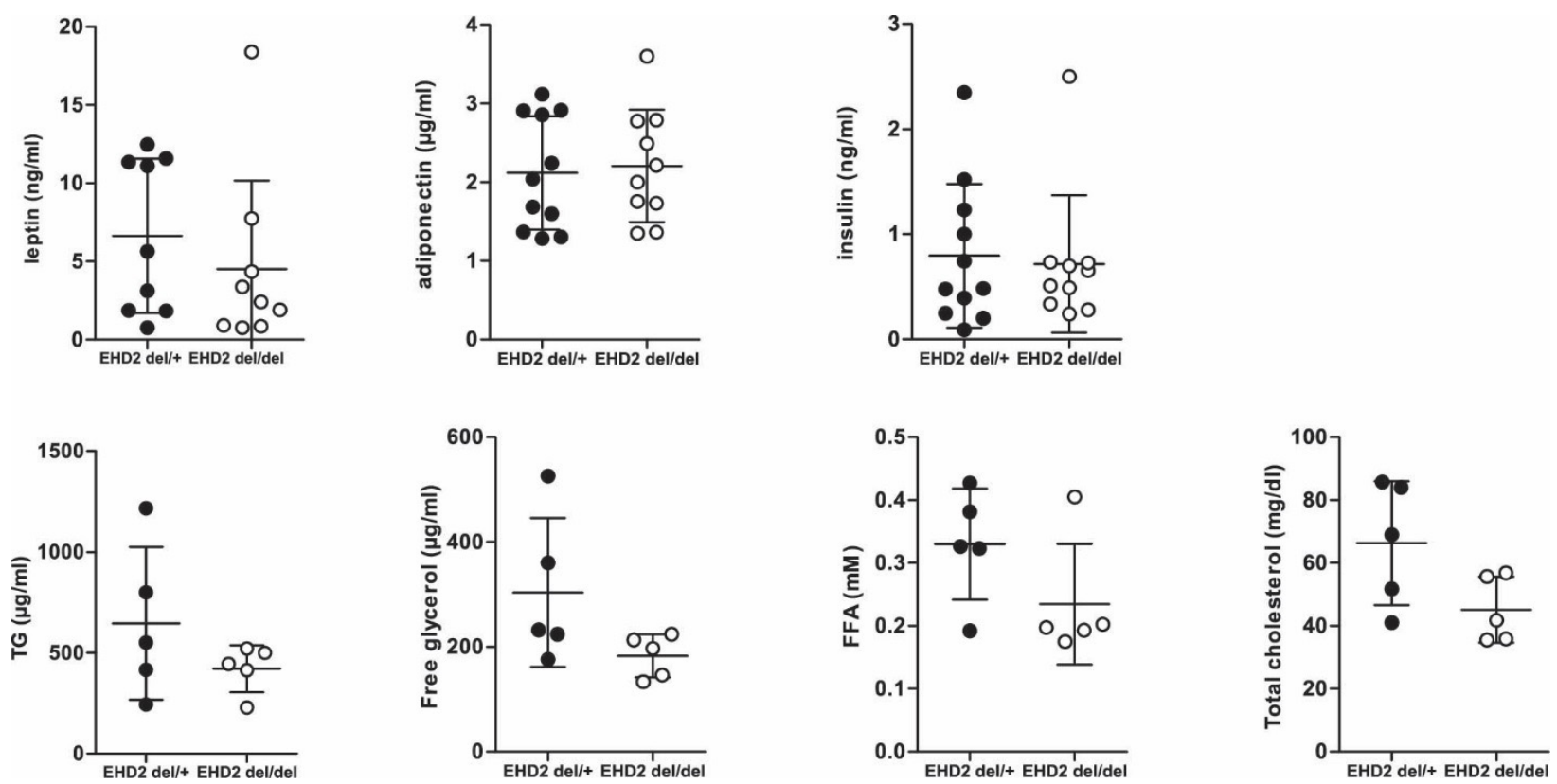

Fig. S3: Blood plasma analysis of EHD2 del/+ and EHD2 del/del mice did not reveal any significant differences (related to Fig. 1)

855 Blood samples obtained from EHD2 del/+ and del/del mice ( $($ EHD2 del/ + ) $=10$ or 5; $n($ EHD2 del/del) = 10 
bioRxiv preprint doi: https://doi.org/10.1101/511709; this version posted March 7, 2019. The copyright holder for this preprint (which was not certified by peer review) is the author/funder, who has granted bioRxiv a license to display the preprint in perpetuity. It is made available under aCC-BY-NC-ND 4.0 International license.
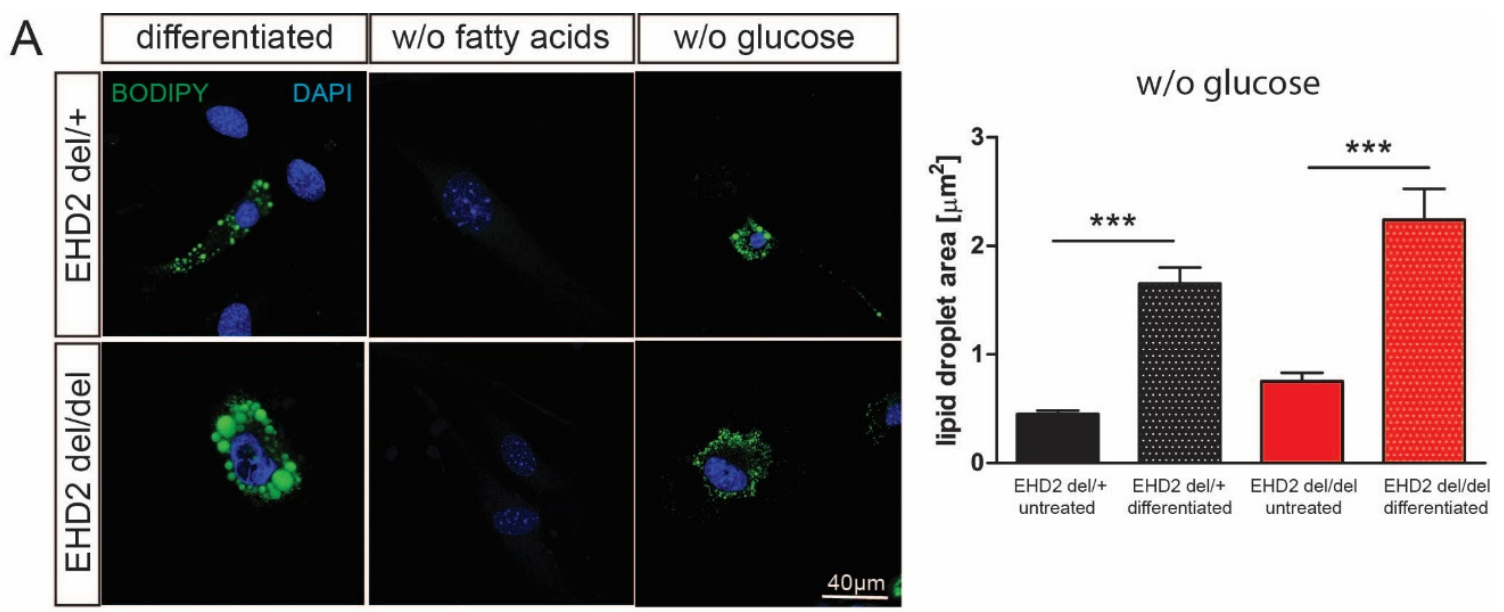

B
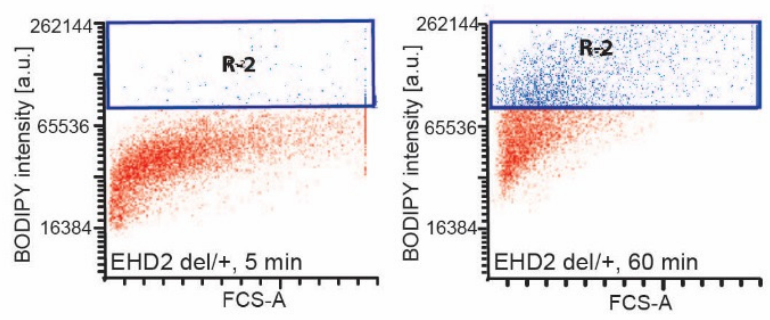

C
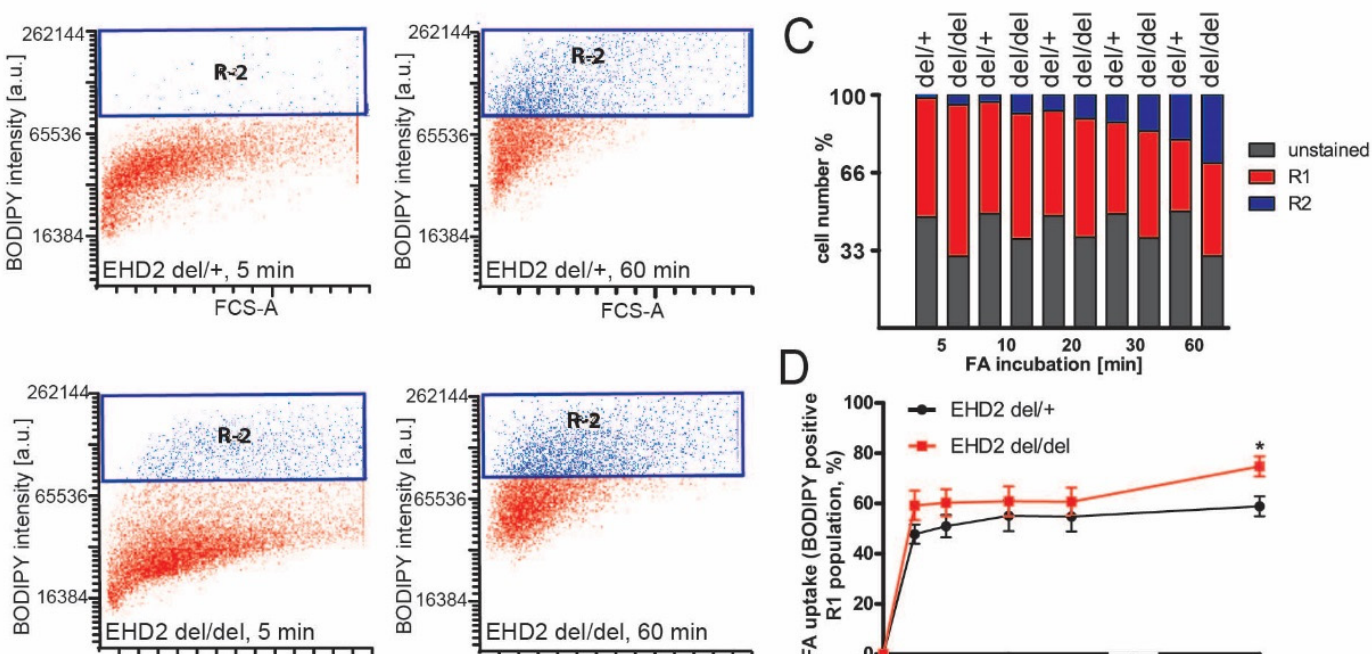

$E$
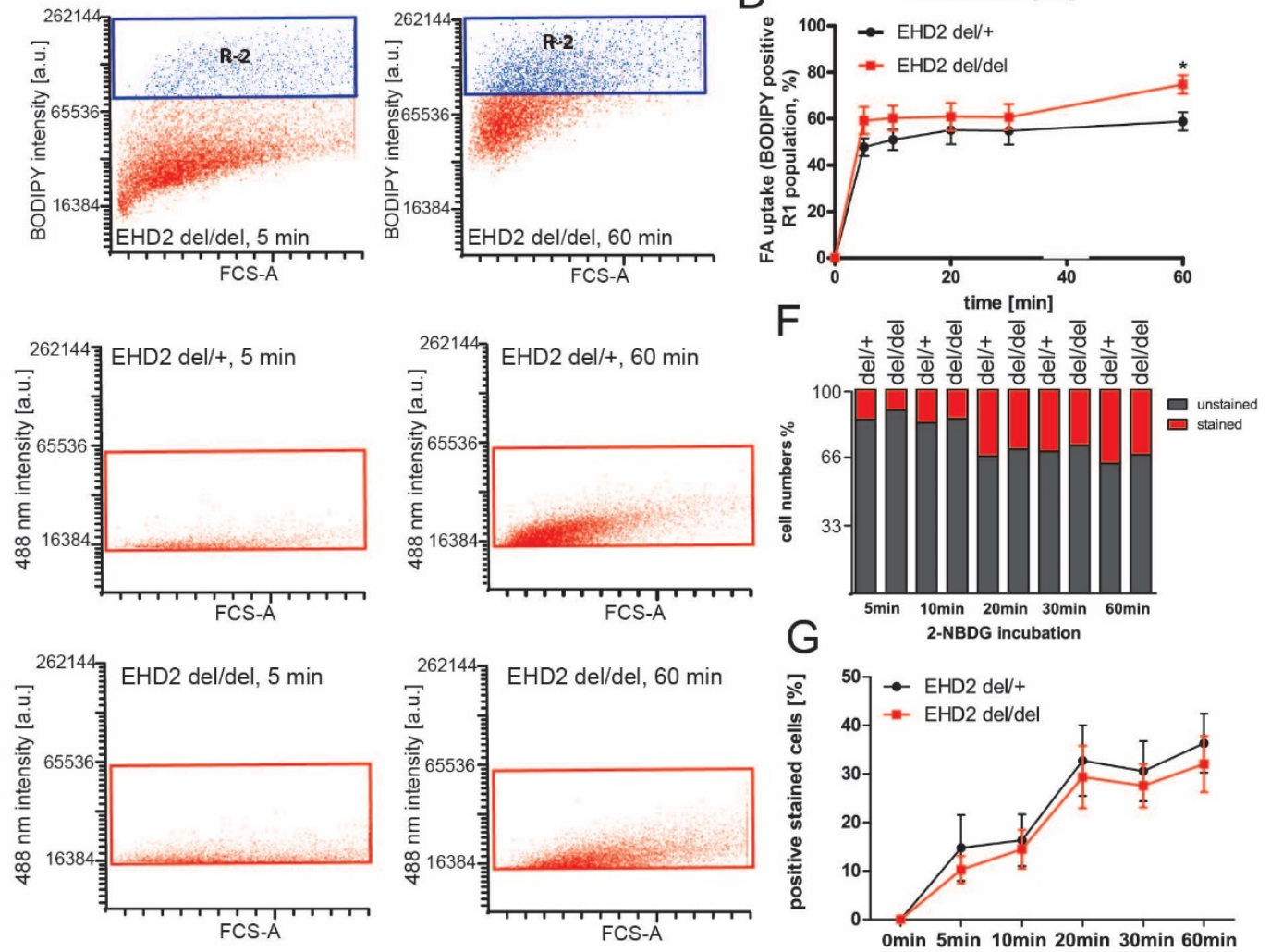

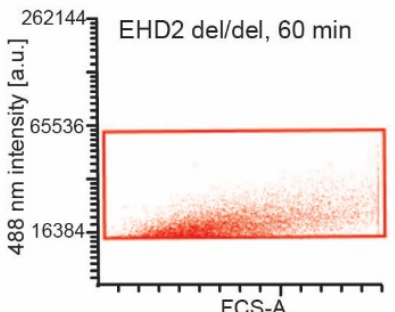

FCS-A $0 \mathrm{~min} 5 \mathrm{~min} 10 \mathrm{~min} 20 \mathrm{~min} 30 \mathrm{~min} 60 \mathrm{~min}$ 


\section{Fig. S4: Cellular investigation of EHD2 del/del adipocytes (related to Fig. 2)}

863 A Pre-adipocytes were treated with either differentiation medium containing delipidated FBS or without 864 glucose and BODIPY staining illustrating LDs.

865 B-D Fatty acid uptake assay in differentiated EHD2 del/+ and EHD2 del/del adipocytes. Dodecanoic acid866 BODIPY uptake was measured after 5, 10, 20, 30 or $60 \mathrm{~min}$, and R1 population indicates positively stained 867 cells (illustrated in red in graphs B, C). R2 populations (blue) correspond to higher BODIPY staining 868 intensity in cells and represent adipocytes with increased amount of dodecanoic acid taken up (shown in 869 blue in graphs B, C). Overview of fatty acid uptake (percent cell numbers (B), and time scale (D); B-D, $870 \mathrm{n}($ del $/+)=6 / 3$ experiments, $\mathrm{n}($ del $/$ del $)=8 / 3$ experiments $)$.

871 E-G Following 5 days of differentiation, glucose uptake in cultured adipocytes was measured after 5-60 $872 \min (C, n=6)$.

873 Line graphs show mean +/- SE, column bar graphs show mean + SE , t-test or Mann Withney U test was 874 used to calculate significance, $* * \mathrm{P}<0.001, * * * \mathrm{P}<0.0001$.

875 
A

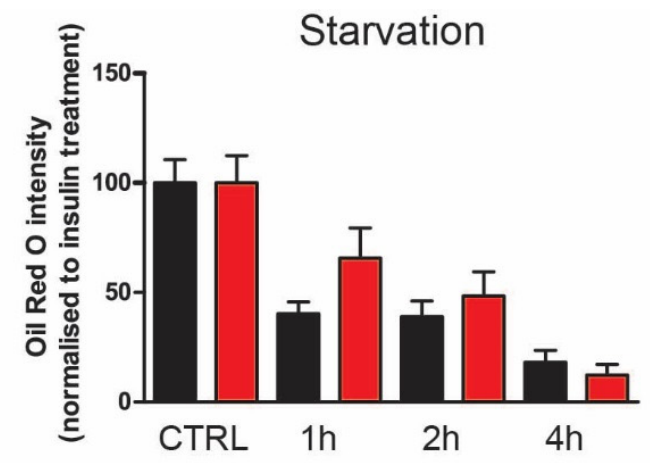

B de novo lipogenesis adipocytes

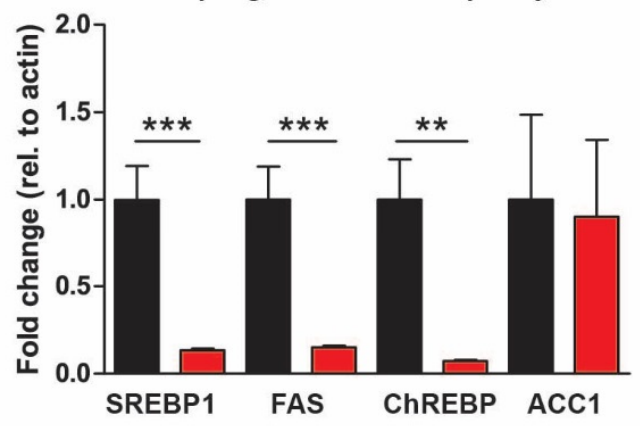

C

- EHD2 del/del

de novo lipogenesis WAT
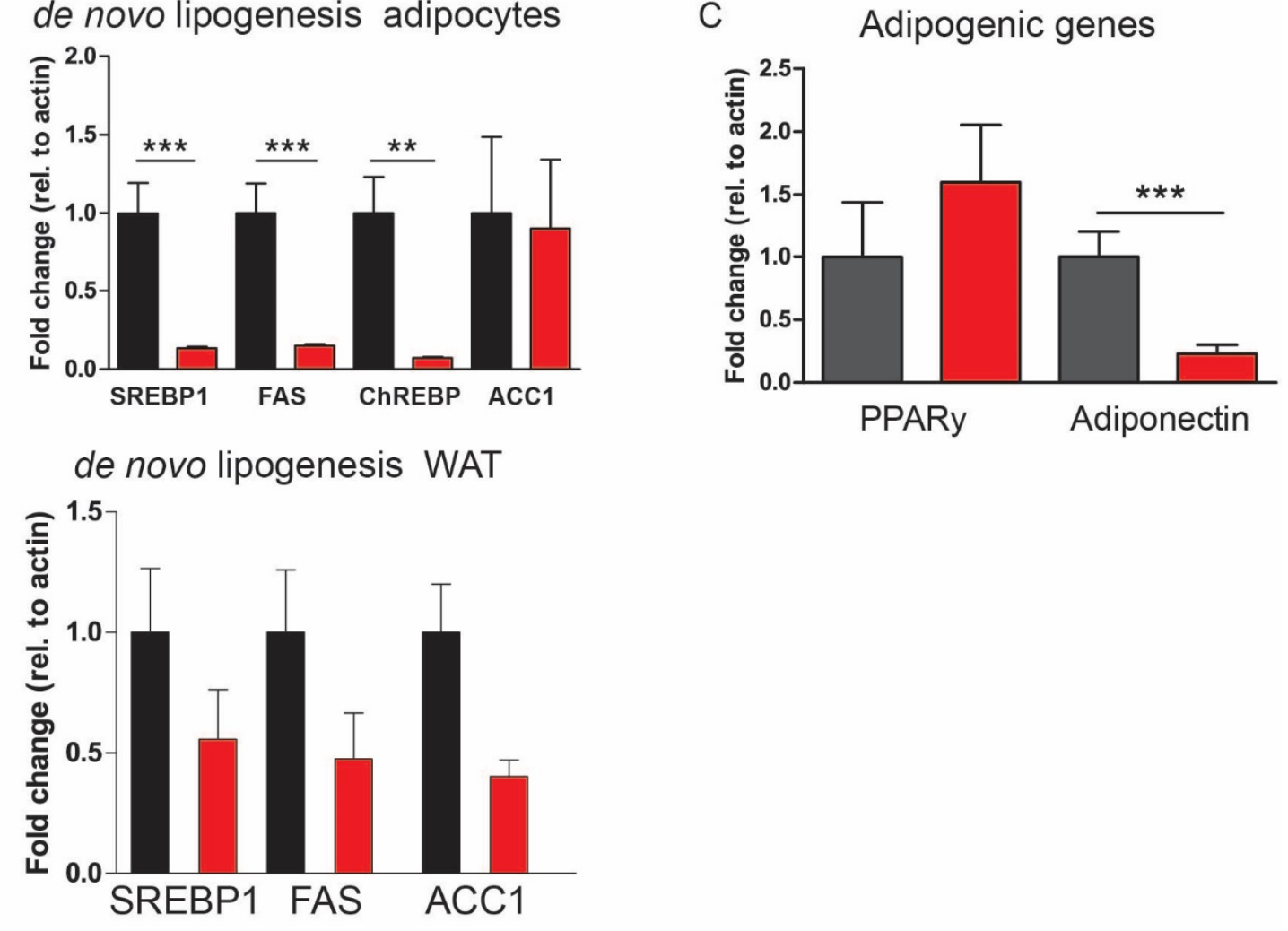

Fig. S5: Cellular investigation of EHD2 del/del adipocytes (related to Fig. 2)

879 A Differentiated EHD2 del/+ and EHD2 del/del adipocytes were starved for 1-4h and Oil Red O staining was applied (n(EHD2 del/ $+=53 / 3 ; n($ EHD2 del $/$ del $)=50 / 3$ ).

881 B-C Gene expression analysis of genes involved in de novo lipogenesis (B) or adipogenic genes (C) in EHD2 del/+ and del/del differentiated adipocytes $(n=8)$.

883 D Gene expression analysis of genes involved in de novo lipogenesis ( $n=5)$.

884 Column bars show mean $+\mathrm{SE}$, t-test or Mann Withney $U$ test was used to calculate significance, ${ }^{* *}$ $\mathrm{P}<0.001, * * * \mathrm{P}<0.0001$. 

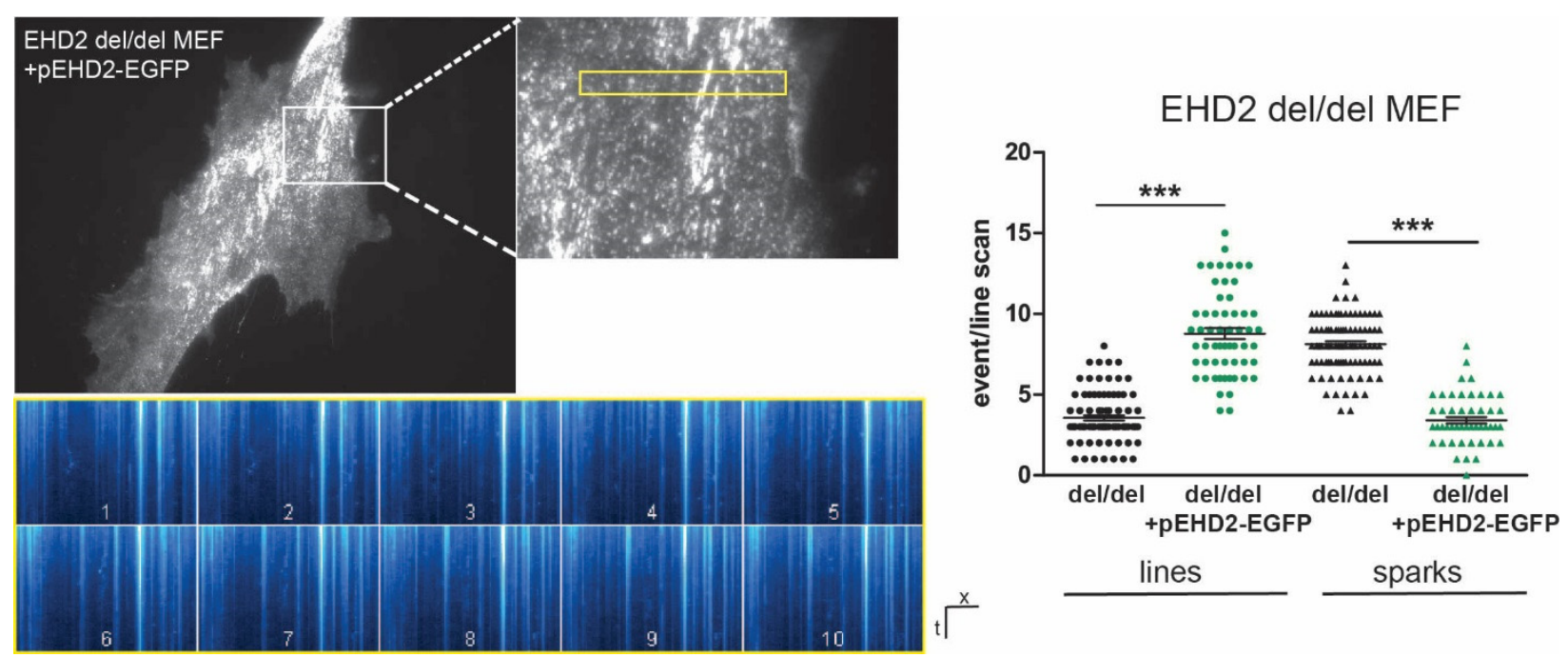

Fig. S6: Caveolar mobility in EHD2 del/del MEFs (related to Fig. 4)

890 TIRF live-imaging in EHD2del/del MEFs transfected with pEHD2-EGFP and pCav1-EGFP (1:1) to investigate 891 single caveolae movement by TIRF live imaging. Non-moving caveolae correspond to vertical lines within the line scan, moving caveolae can be related to single sparks $(B, n=30$, graph illustrates each replicate with mean $+/$ - SE, t-test was used to calculate significance). See also Movie S4. Plots indicate each replicate from maximal to minimum value with mean, $t$-test or Mann Withney $U$ test were used to calculate significance, $* * * \mathrm{P}<0.0001$. 


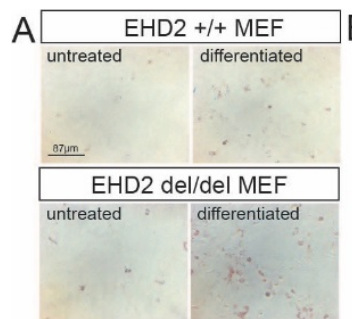

Oil Red $O$ staining after 5 days

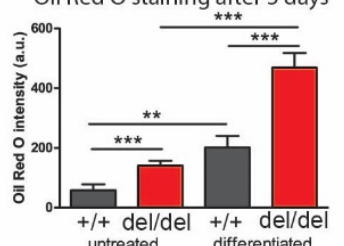

untreated differentiated
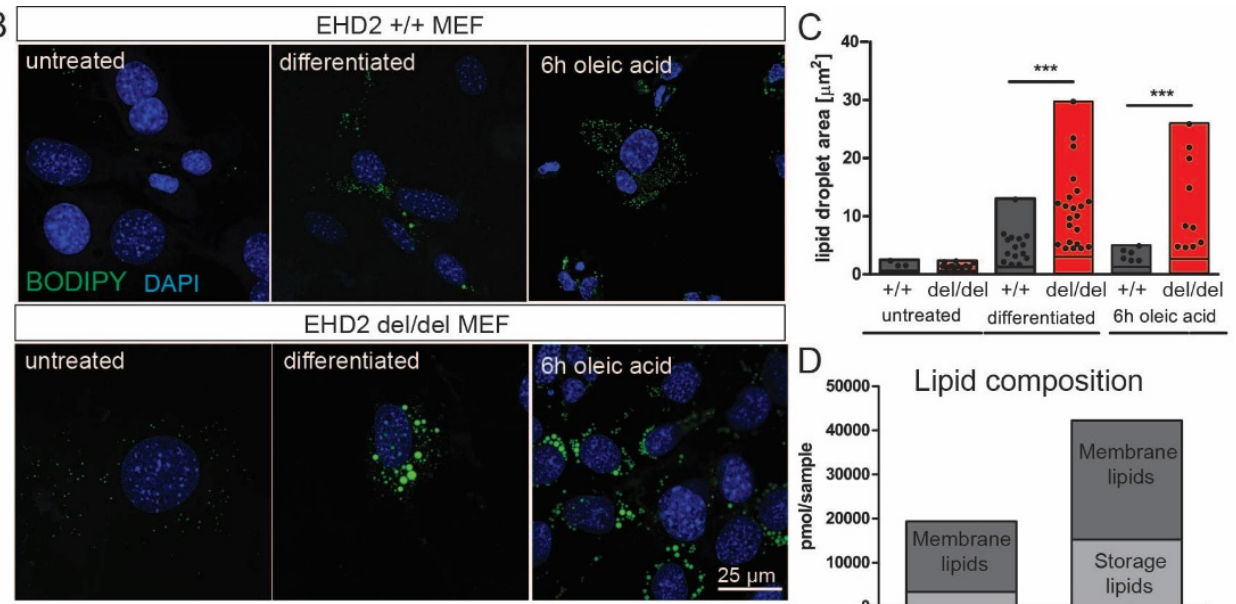

untreated differentiated $6 \mathrm{~h}$ oleic acid

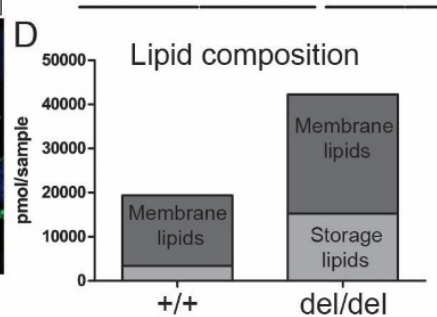

E pEGFP

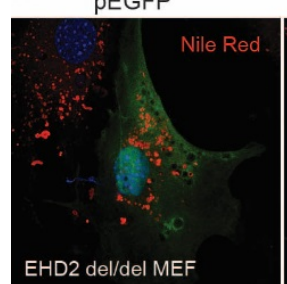

pEHD2-deltaN-EGFP

pEHD2-deltaEH-EGFP
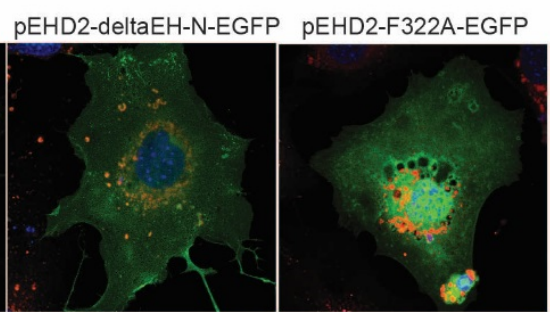

pEHD2-F122A-EGFP

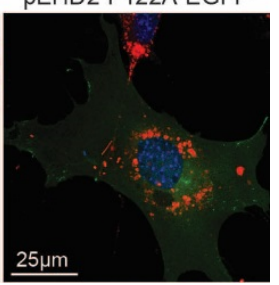

Fig. S7: Caveolar mobility in EHD2 del/del MEFs (related to Fig. 5)

A Oil Red O staining of EHD2 $+/+$ and del/del MEFs untreated or treated with adipocyte differentiation medium (untreated: $\mathrm{n}(+/+)=21 / 3, \mathrm{n}(\mathrm{del} / \mathrm{del})=28 / 4)$; differentiated: $\mathrm{n}(+/+)=37 / 3, \mathrm{n}(\mathrm{del} / \mathrm{del})=55 / 4$ ).

B-C LD analysis after 5 days of differentiation or $6 \mathrm{~h}$ of oleic acid in EHD2 MEFs by BODIPY staining (D, untreated: $n(+/+)=61 / 2, n($ del/del) $=139 / 4)$; differentiated: $n(+/+)=148 / 3, n($ del $/$ del $)=200 / 4$; oleic acid: $\mathrm{n}(+/+)=146 / 3, \mathrm{n}(\mathrm{del} / \mathrm{del})=217 / 3)$.

D Lipid composition analysis of MEFs differentiated for 5 days, 120,000 cells were analyzed for each experiment $(n=3)$.

E EHD2 del/del MEFs were transfected with different EHD2 constructs and lipid droplet size was analyzed after $6 \mathrm{~h}$ oleic acid treatment.

Box plots indicate each replicate from maximal to minimum value with mean, column bar graphs show mean $+\mathrm{SE}$, t-test or Mann Withney $\mathrm{U}$ test were used to calculate significance, ${ }^{* *} \mathrm{P}<0.001, * * * \mathrm{P}<0.0001$. 


\section{$918 \quad$ References SI}

919 1. Cham BE, Knowles BR (1976) A solvent system for delipidation of plasma or serum without $920 \quad$ protein precipitation. J Lipid Res 17:176-181.

921 2. Mehlem A, Hagberg CE, Muhl L, Eriksson U, Falkevall A (2013) Imaging of neutral lipids by oil red 922 O for analyzing the metabolic status in health and disease. Nat Protoc 8(6):1149-1154.

923 3. Kärgel E, et al. (1996) Candida maltosa NADPH-cytochrome P450 reductase: cloning of a fulllength cDNA, heterologous expression in Saccharomyces cerevisiae and function of the Nterminal region for membrane anchoring and proliferation of the endoplasmic reticulum. Yeast

927 4. Wilkinson DD (1993) In situ Hybridization. A Practical Approach. Genet Res 61(03):234.

928 5. Dubikovskaya E, Chudnovskiy R, Karateev G, Park HM, Stahl A (2014) Measurement of long-chain 929 fatty acid uptake into adipocytes. Methods Enzym 538(1):107-134. 